R. Denk $\cdot$ M. Faierman · M. Möller

\title{
An elliptic boundary problem for a system involving a discontinuous weight
}

\begin{abstract}
In a recent paper, Agranovich, Denk and Faierman dealt with a priori estimates, completeness, Abel-Lidskii summability, and eigenvalue asymptotics for scalar elliptic boundary eigenvalue problems involving discontinuous weights. Here we extend these results to the matrix valued case with a diagonal discontinuous weight matrix. The given region is subdivided into subregions on which the weights are continuous. Whereas in the scalar case the usual ellipticity conditions suffice to obtain a priori estimates, a counterexample shows that here transmission conditions at the boundaries of the subregions are also needed.
\end{abstract}

\section{Introduction}

The object of this paper is to extend the results of [6] for a scalar boundary problem involving a discontinuous weight function to that for a system. Accordingly, we shall be concerned here with the boundary problem

$$
\begin{aligned}
A(x, D) u(x)-\lambda \omega(x) u(x) & =f(x) \text { in } \Omega \\
B_{j}(x, D) u(x) & =g_{j}(x) \text { on } \Gamma \text { for } j=1, \ldots, m N,
\end{aligned}
$$

where $\Omega$ is a bounded region in $\mathbb{R}^{n}, n \geq 2$, with boundary $\Gamma, m, N \in \mathbb{N}$ with $N \geq 2, u(x)=\left(u_{1}(x), \ldots, u_{N}(x)\right)^{T}$ and $f(x)=\left(f_{1}(x), \ldots, f_{N}(x)\right)^{T}$ are $N \times 1$ matrix functions defined in $\Omega,{ }^{T}$ denotes transpose, the $g_{j}(x)$ are scalar functions defined on $\Gamma, A(x, D)$ is an $N \times N$ matrix operator whose entries $A_{j k}(x, D)$ are linear differential operators defined on $\Omega$ of order not exceeding $2 m, B_{j}(x, D), 1 \leq j \leq m N$, is a $1 \times N$ matrix operator whose entries $B_{j k}(x, D)$ are linear differential operators defined on $\Gamma$ of order not exceeding $m_{j}<2 m$, and $\omega(x)=\operatorname{diag}\left(\omega_{1}(x), \ldots, \omega_{N}(x)\right)$ with the $\omega_{j}(x)$ being real-valued functions in $L_{\infty}(\Omega)$. Our assumptions concerning the problem (1.1), (1.2) will be made precise in Section 2.

Let us mention at this point some of our assumptions concerning the problem (1.1), (1.2). We suppose that there exists the sequence $\left\{\Omega_{r}\right\}_{1}^{N_{0}}, N_{0} \in \mathbb{N}$, of

R. Denk: NWF I-Mathematik, Universität Regensburg, D-93040 Regensburg, Germany

M. Faierman: School of Mathematics, University of the Witwatersrand, WITS, 2050, South Africa, e-mail: 036mef@ cosmos.wits.ac.za

M. Möller: School of Mathematics, University of the Witwatersrand, WITS, 2050, South Africa, e-mail: 036man@ cosmos.wits.ac.za

Konstanzer Online-Publikations-System (KOPS)

URL: http://www.ub.uni-konstanz.de/kops/volltexte/2008/5065/

URN: http://nbn-resolving.de/urn:nbn:de:bsz:352-opus-50653 
non-empty subregions of $\Omega$ whose closures are also contained in $\Omega$ and pairwise disjoint. Furthermore, if we let $\Omega_{0}=\Omega \backslash \bigcup_{r=1}^{N_{0}} \overline{\Omega_{r}}$, then we suppose that for each $r, 0 \leq r \leq N_{0}$, the $\omega_{j}(x)$ are continuous in $\Omega_{r}$ and extend by continuity to continuous non-vanishing functions in $\overline{\Omega_{r}}$. Thus we allow $\omega(x)$ to have discontinuities at the boundaries $\Gamma_{r}$ of the $\Omega_{r}, r=1, \ldots, N_{0}$.

Under our given assumptions we shall proceed as in [6]. Firstly we shall establish our basic theorem, Theorem 2.4, concerning the existence and uniqueness of and a priori estimates for solutions of the boundary problem (1.1), (1.2) in an $L_{p}$ Sobolev space setting $(1<p<\infty)$. Then turning to the spectral problem associated with (1.1), (1.2), we let $A_{p}$ denote the operator induced in $L_{p}(\Omega)^{N}$ by (1.1) and the boundary conditions (1.2) with the $g_{j}=0$, let $V_{p}$ denote the operator of multiplication induced in $L_{p}(\Omega)^{N}$ by $\omega(x)$, and interpret the spectral problem (1.1), (1.2) (with the $g_{j}=0$ ) as the spectral problem for the pencil $A_{2}-\lambda V_{2}$ acting in $L_{2}(\Omega)^{N}$, or equivalently, as the spectral problem for the operator $V_{2}^{-1} A_{2}$ acting in $L_{2}(\Omega)^{N}$. By appealing to our basic theorem, we shall then derive results concerning completeness and Abel-Lidskii summability of the principal vectors of $V_{2}^{-1} A_{2}$ in various function spaces as well as concerning the angular distribution of its eigenvalues. Finally, by appealing again to our basic theorem, we shall derive rough and principal asymptotic estimates for the eigenvalues of $V_{2}^{-1} A_{2}$.

We have just seen that all the results derived here concerning the spectral properties of the problem (1.1), (1.2) depend upon our basic theorem, Theorem 2.4. However, with regards to Theorem 2.4, certain differences arise between the scalar case considered in [6] and the matrix case considered here. To indicate these differences let us mention some of the conditions required for the validity of Theorem 2.4. Firstly we impose the condition that there exists the closed sector $\mathcal{L}$ in the $\lambda$-plane with vertex at the origin such that for $r=0, \ldots, N_{0}$,

$$
\operatorname{det}(\stackrel{\circ}{A}(x, \xi)-\lambda \omega(x)) \neq 0 \text { for }(x, \xi) \in \overline{\Omega_{r}} \times \mathbb{R}^{n} \text { and } \lambda \in \mathcal{L} \text { if }|\xi|+|\lambda| \neq 0,
$$

where $\AA(x, \xi)$ denotes the principal symbol of $A(x, D)$. Secondly we impose the condition that the system of boundary operators $\left\{B_{j}(x, D)\right\}_{1}^{m N}$ covers the operator $A(x, D)-\lambda \omega(x)$ on $\Gamma$ in the sense that Condition 2 of Definition 2.3 is satisfied. Then apart from certain smoothness assumptions concerning the operators and boundaries involved, these two conditions suffice for the validity of Theorem 2.4 in the scalar case, but not for the matrix case considered here. To clarify this statement, let us mention again that in this work we treat the problem (1.1), (1.2) in a Sobolev space setting, namely in the space $u \in W_{p}^{2 m}(\Omega)^{N}$. Hence, because of the discontinuities of $\omega(x)$ at the boundaries $\Gamma_{r}$, the boundary problem (1.1), (1.2) is in fact to be interpreted as the boundary problem: (1.1a) (which is obtained from (1.1) by replacing $\Omega$ there by $\left.\stackrel{\circ}{\Omega}=\bigcup_{r=0}^{N_{0}} \Omega_{r}\right),(1.2)$, and

$$
B_{j, \ell}^{(r)}(x, D) u(x)=0 \text { on } \Gamma_{r} \text { for } j=1, \ldots, N \text { and } \ell=0, \ldots,(2 m-1),
$$

$r=1, \ldots, N_{0}$, where $B_{j, \ell}^{(r)}(x, D)=\partial_{\nu}^{\ell} u_{j}^{(r)}(x)-\partial_{\nu}^{\ell} u_{j}^{(0)}(x), u^{(r)}(x)=u(x) \mid \Omega_{r}$ for $r=0, \ldots, N_{0}$, and $\partial_{\nu}$ denotes differentiation along the interior normal to $\Gamma_{r}$. Then the third condition we require for the validity of Theorem 2.4 is that the 
system of boundary operators (1.4) covers the operator $A(x, D)-\lambda \omega(x)$ on $\Gamma_{r}$ for $r=1, \ldots, N_{0}$ in the sense that Condition 3 of Definition 2.3 is satisfied. However for the scalar case it is shown in [6, Section 7] that our third condition is redundant, i.e., the boundary operators (1.4) are absolutely elliptic in the sense of [2], while on the other hand this is certainly not the case for the matrix problem considered here, as we shall show by means of an example (see Example A.4 of Appendix A). Thus in the matrix case our third condition imposes a restriction upon the set $(A(x, D), \omega(x))$ for which the first two conditions cited above are satisfied. One important case where the first two conditions suffice for the validity of Theorem 2.4 occurs when $\stackrel{\circ}{A}(x, \xi), \xi \in \mathbb{R}^{n} \backslash\{0\}$, is positive definite at all points of $\Gamma_{r}$, $r=1, \ldots, N_{0}$ (see Proposition A.1). Another such case occurs when the matrix operator $\stackrel{\circ}{A}(x, D)$ is triangular at all points of $\Gamma_{r}, r=1, \ldots, N_{0}$ (see Proposition A.3).

To indicate a further difference between the scalar problem considered in [6] and the matrix problem considered here, let us point out that in this work only limited smoothness assumptions are made at first concerning the operators and boundaries involved (see Condition (a) of Definition 2.2). These smoothness conditions, together with the conditions cited above, ensure the validity of our basic theorem. However the basic theorem alone does not suffice in allowing us to arrive at the eigenvalue asymptotics for the problem (1.1), (1.2). Indeed, in order to achieve this end, we will employ the method of [6] for obtaining eigenvalue asymptotics under limited smoothness assumptions. However this method requires not only the validity of Theorem 2.4 , but requires as well that the boundary problem formally adjoint to (1.1), (1.2),

$$
\begin{aligned}
A^{\prime}(x, D) u(x)-\bar{\lambda} \omega(x) u(x) & =f(x) \text { in } \Omega, \\
B_{j}^{\prime}(x, D) u(x) & =g_{j}(x) \text { on } \Gamma \text { for } j=1, \ldots, m N,
\end{aligned}
$$

is well defined and that the analogue of Theorem 2.4 for the problem (1.5), (1.6) holds also. Here $A^{\prime}(x, D)$ is the formal adjoint of $A(x, D)$ and $B_{j}^{\prime}(x, D), 1 \leq$ $j \leq m N$, is a $1 \times N$ matrix operator whose entries are linear differential operators defined on $\Gamma$ of order not exceeding $m_{j}^{\prime}<2 m$ (see Proposition A.6). Thus in this paper we shall impose further conditions (see Condition (b) of Definition 2.2) which will ensure that the problem (1.5), (1.6) is well defined and that $A^{\prime}(x, D)$ and the $B_{j}^{\prime}(x, D)$ satisfy the same smoothness assumptions as do $A(x, D)$ and the $B_{j}(x, D)$ (see Proposition A.6). Then turning to the validity of the basic theorem for (1.5), (1.6), it is clear that the first two conditions cited above for the problem (1.1), (1.2) imply their analogues for the problem (1.5), (1.6). The third condition that we require is that the system of boundary operators (1.4) covers the operator $A^{\prime}(x, D)-\bar{\lambda} \omega(x)$ on $\Gamma_{r}$ for $r=1, \ldots, N_{0}$. For the scalar problem considered in [6] this condition is redundant since the boundary operators (1.4) are absolutely elliptic, while for the problem considered here this condition appears to impose a further restriction upon the $A(x, D)$ and $\omega(x)$ which can be dealt with according to our theory. However we shall show in Appendix A (see Proposition A.5) that this is not the case. Indeed, we shall prove that if the system of boundary operators (1.4) covers the operator $A(x, D)-\lambda \omega(x)$ on $\Gamma_{r}\left(1 \leq r \leq N_{0}\right)$, then it also 
covers the operator $A^{\prime}(x, D)-\bar{\lambda} \omega(x)$ on $\Gamma_{r}$. It is precisely here where the further difference between the scalar and matrix problems appear; for in proving this result we require stronger smoothness assumptions concerning the $\Gamma_{r}$ than those supposed in [6] (see the first paragraph of Subsection 8.1 of [6]).

It is of interest to compare our problem to a similar one considered by Sango [17], [18] wherein the spectral properties of the boundary problem (1.1a), (1.2), (1.4) have been investigated. By appealing to the results of [6], Sango derives information concerning this problem similar to those cited above for our problem. However, there are points in [18], and in particular, one which pertains to the treatment of the adjoint problem (the significance of which has already been pointed out in the previous paragraph), which are questionable. Let us fix our attention now upon this last point. Assuming only smoothness assumptions which are sufficient to establish the basic theorem (Theorem 2.4) for the system (1.1), (1.2), but certainly not sufficient to even construct the formal adjoint $A^{\prime}(x, D)$ of $A(x, D)$, Sango deals with the adjoint problem by introducing the Assumption $*$, which consists of two parts. In the first part he requires that the boundary operators $\left\{B_{j}(x, D)\right\}_{1}^{m N}$ are normal (see [9], [13, Definition 1.4.3, p.50], [16]). However we shall show in the sequel that Sangos' earlier assumptions imply normality, and hence this first part is redundant. In the second part Sango requires that the boundary problem formally adjoint to (1.1a), (1.2), (1.4): (1.5a) (which is obtained from (1.5) by replacing $\Omega$ by $\Omega$ ), (1.6), (1.4) is well defined and satisfies at least those smoothness assumptions as were imposed upon the problem (1.1), (1.2) to ensure the validity of the basic theorem. Then he states that the boundary operators (1.4) are not the adjoint boundary operators for the $\Gamma_{r}$ appearing in the formal adjoint problem of (1.1a), (1.2), (1.4), but claims that if one approaches the problem from local considerations, then they are (of course this does not make sense unless we take it that he means that the two systems of boundary operators are equivalent in the sense of [15, p.121]). Sango gives no proof of this claim, but states that it is proved in [9]. However, we find all of the second part of Assumption $*$ unacceptable for the following reasons. Firstly, Sango gives no indication at all of the smoothness assumptions required for the validity of the basic theorem for the adjoint problem (1.5), (1.6). Secondly, and most importantly, Sango's assertion that his claim is proved in [9] is false since the result referred to there deals only with boundary operators whose orders do not exceed $m-1$, while the boundary operators Sango has to deal with have orders up to $2 m-1$. Thus, in light of this unproved claim and from a scrutiny of the proofs of his various theorems, one is left in doubt as to whether Sangos' results concerning the eigenvalue asymptotics are correct.

Finally, in Section 2 of this paper we introduce those assumptions and definitions which we require for our work as well as prove the basic theorem, Theorem 2.4. In Section 3 we prove those results cited above concerning completeness, Abel-Lidskii summability, and the angular distribution of the eigenvalues. Section 4 is devoted to the eigenvalue asymptotics, and in Appendix A we prove the various assertions that have been made above. 


\section{Preliminaries}

In this section we are going to introduce some assumptions and definitions which we require for our work as well as prove the basic theorem, Theorem 2.4. Hence to begin with, we let $x=\left(x_{1}, \ldots, x_{n}\right)=\left(x^{\prime}, x_{n}\right)$ denote a generic point in $\mathbb{R}^{n}$ and use the notation $D_{j}=-i \partial / \partial x_{j}, D=\left(D_{1}, \ldots, D_{n}\right)=\left(D^{\prime}, D_{n}\right), D^{\alpha}=$ $D_{1}^{\alpha_{1}} \cdots D_{n}^{\alpha_{n}}=D^{\prime \alpha^{\prime}} D_{n}^{\alpha_{n}}, \xi^{\alpha}=\xi_{1}^{\alpha_{1}} \cdots \xi_{n}^{\alpha_{n}}=\xi^{\prime \alpha^{\prime}} \xi_{n}^{\alpha_{n}}$ for $\xi=\left(\xi_{1}, \ldots, \xi_{n}\right)=$ $\left(\xi^{\prime}, \xi_{n}\right) \in \mathbb{R}^{n}$, where $\alpha=\left(\alpha_{1}, \ldots, \alpha_{n}\right)=\left(\alpha^{\prime}, \alpha_{n}\right)$ is a multi-index whose length $\sum_{j=1}^{n} \alpha_{j}$ is denoted by $|\alpha|$. We also let $\mathbb{R}_{ \pm}^{n}=\left\{x \in \mathbb{R}^{n} \mid x_{n} \gtrless 0\right\}$ (in the sequel we shall at times use the notation $\mathbb{R}_{ \pm}$whose meaning is clear). For $1<p<\infty$, $0 \leq s<\infty, \ell \in \mathbb{N}$, and $G$ an open set in $\mathbb{R}^{\ell}$, we let $W_{p}^{s}(G)$ denote the usual Sobolev space of order $s$ related to $L_{p}(G)$ and let $\|\cdot\|_{s, p, G}$ denote the norm in this space.

Of particular interest to us is the case where $G$ is an open bounded set in $\mathbb{R}^{n}$ and its boundary, $\partial G$, is of class $C^{2 m-1,1}$. For this case we can consider $\partial G$ as a $C^{2 m-1,1}$ submanifold of $\mathbb{R}^{n}$ of codimension 1 . For $s$ an integer satisfying $1 \leq s \leq 2 m$, for $\left\{\left(U_{j}, \kappa_{j}\right)\right\}_{1}^{\ell}$ a $C^{2 m-1,1}$ atlas for $\partial G$, and for $\left\{\phi_{j}\right\}_{1}^{\ell}$ a $C^{2 m-1,1}$ partition of unity subordinate to the open covering $\left\{U_{j}\right\}_{1}^{\ell}$ of $\partial G$, we define (see $[11$, p.20], [15, p.35])

$$
W_{p}^{s-1 / p}(\partial G)=\left\{v \in \mathcal{D}^{\prime}(\partial G) \mid\left(\phi_{j} v\right) \circ \kappa_{j}^{-1} \in W_{p}^{s-1 / p}\left(\mathbb{R}^{n-1}\right), j=1, \ldots, \ell\right\} .
$$

It can be shown that the definition (2.1) is independent of the atlas $\left\{\left(U_{j}, \kappa_{j}\right)\right\}_{1}^{\ell}$ and the partition of unity $\left\{\phi_{j}\right\}_{1}^{\ell}$. If we equip $W_{p}^{s-1 / p}(\partial G)$ with the norm

$$
\|v\|_{s-1 / p, p, \partial G}=\sum_{j=1}^{\ell}\left\|\left(\phi_{j} v\right) \circ \kappa_{j}^{-1}\right\|_{s-1 / p, p, \mathbb{R}^{n-1}},
$$

then $W_{p}^{s-1 / p}(\partial G)$ is a Banach space, and we can also show that different atlases and partitions of unity give rise to equivalent norms. We can also characterize $W_{p}^{s-1 / p}(\partial G)$ as the space of boundary values $v=u \mid \partial G$ of the vectors $u \in W_{p}^{s}(G)$, and if we equip $W_{p}^{s-1 / p}(\partial G)$ with the norm

$$
\|v\|_{s-1 / p, p, \partial G}^{\dagger}=\inf \|u\|_{s, p, G},
$$

where the infimum is taken over all $u \in W_{p}^{s}(G)$ satisfying $u_{\left.\right|_{\partial G}}=v$, then the norms (2.2) and (2.3) are equivalent.

It will be convenient for us to include a parameter in the norms defined above, namely for $\lambda \in \mathbb{C}$ we set

$$
\begin{aligned}
\|u \mid\|_{s, p, G} & =\|u\|_{s, p, G}+|\lambda|^{s / 2 m}\|u\|_{0, p, G} \text { for } u \in W_{p}^{s}(G), \\
\|\| v \|_{s-1 / p, p, \partial G} & =\|v\|_{s-1 / p, p, \partial G}+|\lambda|^{(s-1 / p) / 2 m}\|v\|_{0, p, \partial G} \text { for } v \in W_{p}^{s-1 / p}(\partial G),
\end{aligned}
$$

where $\|\cdot\|_{0, p, \partial G}$ denotes the norm in $L_{p}(\partial G)$.

At times in the sequel we should have to deal with the space $W_{p}^{s}(G)^{N}$; and we shall equip this space with either of the norms \|\|$\cdot\left\|\left.\right|_{W_{p}^{s}(G)^{N}},\right\| \cdot\|\|_{W_{p}^{s}(G)^{N}}$, where 
\|\|$u\|\|_{W_{p}^{s}(G)^{N}}=\left(\sum_{j=1}^{N}\|\| u_{j} \|_{s, p, G}^{p}\right)^{1 / p},\|u\|_{W_{p}^{s}(G)^{N}}=\left(\sum_{j=1}^{N}\left\|u_{j}\right\|_{s, p, G}^{p}\right)^{1 / p}$
for $u=\left(u_{1}, \ldots, u_{n}\right)^{T} \in W_{p}^{s}(G)^{N}$.

Let us now turn to the boundary problem (1.1), (1.2).

Assumption 2.1. We suppose henceforth that all the assertions made in the second paragraph of Section 1 concerning the $\Omega_{r}$ and $\omega(x)$ hold.

Next let us write

$$
\begin{aligned}
& A_{j k}(x, D)=\sum_{|\alpha| \leq 2 m} a_{\alpha}^{j k}(x) D^{\alpha} \text { for } x \in \Omega \text { and } 1 \leq j, k \leq N, \\
& B_{j k}(x, D)=\sum_{|\alpha| \leq m_{j}} b_{\alpha}^{j k}(x) D^{\alpha} \text { for } x \in \Gamma \text { and } k=1, \ldots, N, j=1, \ldots, m N .
\end{aligned}
$$

\section{Definition 2.2.}

(a) Minimal smoothness. The boundary problem (1.1), (1.2) will be called minimally smooth if: (1) $\Gamma$ and the $\Gamma_{r}$ are of class $C^{2 m-1,1}$; (2) for each pair $j, k, a_{\alpha}^{j k} \in L_{\infty}(\Omega)$ for $|\alpha| \leq 2 m$, while the top order coefficients $a_{\alpha}^{j k}(x)$ $(|\alpha|=2 m)$ are continuous in $\bar{\Omega}$; (3) for each pair $j, k, b_{\alpha}^{j k} \in C^{2 m-m_{j}-1,1}(\Gamma)$ for $|\alpha| \leq m_{j}$.

(b) Weak smoothness. The boundary problem (1.1), (1.2) will be called weakly smooth if: (1) $\Gamma$ and the $\Gamma_{r}$ are of class $C^{2 m, 1}$; (2) for each pair $j, k, a_{\alpha}^{j k} \in$ $C^{|\alpha|-1,1}(\bar{\Omega})$ for $1 \leq|\alpha| \leq 2 m, a_{\alpha}^{j k} \in L_{\infty}(\Omega)$ otherwise; (3) for each pair $j, k, b_{\alpha}^{j k} \in C^{2 m-m_{j}-1,1}(\Gamma) \cap C^{|\alpha|, 1}(\Gamma)$ for $|\alpha| \leq m_{j}$.

For $\xi \in \mathbb{R}^{n}$ let

$$
\begin{aligned}
& \stackrel{\circ}{A}(x, \xi)=\left(\stackrel{\circ}{A}_{j k}(x, \xi)\right)_{j, k=1}^{N} \text { for } x \in \bar{\Omega}, \\
& \stackrel{\circ}{B}(x, \xi)=\left(\stackrel{\circ}{B}_{j k}(x, \xi)\right)_{\substack{j=1, \ldots, m N \\
k=1, \ldots, N}} \text { for } x \in \Gamma,
\end{aligned}
$$

and denote by $\stackrel{\circ}{B}_{j}(x, \xi)$ the $j$-th row of $\stackrel{\circ}{B}(x, \xi)$, where $\stackrel{\circ}{A}_{j k}(x, \xi)$ and $\stackrel{\circ}{B}_{j k}(x, \xi)$ denote the principal symbols of $A_{j k}(x, D)$ and $B_{j k}(x, D)$ respectively.

Definition 2.3. Let $\mathcal{L}$ be a closed sector in the complex plane with vertex at the origin. Then the problem (1.1), (1.2) will be called elliptic with parameter in $\mathcal{L}$ if it is minimally smooth and the following conditions are satisfied.

1. (1.3) holds for $r=0, \ldots, N_{0}$.

2. Let $x^{0} \in \Gamma$. Assume that the boundary problem (1.1), (1.2) is rewritten in terms of the local coordinates at $x^{0}$ (here $x^{0} \rightarrow 0$ and $\nu \rightarrow e_{n}$, where $\nu$ is the interior normal to $\Gamma$ at $x^{0}$ and $\left(e_{1}, \ldots, e_{n}\right)$ denotes the standard basis in $\left.\mathbb{R}^{n}\right)$. Then the boundary problem on the half-line

$$
\begin{aligned}
& \stackrel{\circ}{A}\left(0, \xi^{\prime}, D_{n}\right) v(t)-\lambda \omega(0) v(t)=0 \text { for } t=x_{n}>0, \\
& \stackrel{\circ}{B}_{j}\left(0, \xi^{\prime}, D_{n}\right) v(t)=0 \text { at } t=0 \text { for } j=1, \ldots, m N,
\end{aligned}
$$




$$
|v(t)| \rightarrow 0 \text { as } t \rightarrow \infty
$$

has only the trivial solution for $\xi^{\prime} \in \mathbb{R}^{n-1}$ and $\lambda \in \mathcal{L}$ if $\left|\xi^{\prime}\right|+|\lambda| \neq 0$.

3. Let $x^{0} \in \Gamma_{r}, 1 \leq r \leq N_{0}$. Assume that the boundary problem (1.1), (1.4) is rewritten in terms of the local coordinates at $x^{0}$ (as explained in Condition 2 above, where we now replace $\Gamma$ by $\Gamma_{r}$ and $\nu$ points into $\Omega_{r}$ ). Then the boundary problem on the line

$$
\begin{gathered}
\stackrel{\circ}{A}\left(0, \xi^{\prime}, D_{n}\right) v^{+}(t)-\lambda \omega^{+}(0) v^{+}(t)=0 \text { for } t=x_{n}>0, \\
\stackrel{\circ}{A}\left(0, \xi^{\prime}, D_{n}\right) v^{-}(t)-\lambda \omega^{-}(0) v^{-}(t)=0 \text { for } t<0, \\
D_{n}^{\ell} v_{j}^{+}(t)-D_{n}^{\ell} v_{j}^{-}(t)=0 \text { at } t=0 \text { for } j=1, \ldots, N \text { and } \ell=0, \ldots,(2 m-1) \\
\left|v^{+}(t)\right| \rightarrow 0 \text { as } t \rightarrow \infty,\left|v^{-}(t)\right| \rightarrow 0 \text { as } t \rightarrow-\infty
\end{gathered}
$$

has only the trivial solution for $\xi^{\prime} \in \mathbb{R}^{n-1}$ and $\lambda \in \mathcal{L}$ if $\left|\xi^{\prime}\right|+|\lambda| \neq 0$, where $\omega^{+}(x)$ (resp. $\omega^{-}(x)$ ) denotes the restriction of $\omega(x)$ to some appropriate subset of $\overline{\mathbb{R}_{+}^{n}}$ (resp. $\overline{\mathbb{R}_{-}^{n}}$ ) and the $v_{j}^{ \pm}(t)$ denote the components of the $v^{ \pm}(t)$.

We now come to our basic theorem.

Theorem 2.4. Suppose that the boundary problem (1.1), (1.2) is elliptic with parameter in the sector $\mathcal{L}$. Then there exists a $\lambda_{0}=\lambda_{0}(p)>0$ such that for $\lambda \in \mathcal{L}$ with $|\lambda| \geq \lambda_{0}$, the boundary problem has a unique solution $u \in W_{p}^{2 m}(\Omega)^{N}$ for any $f \in L_{p}(\Omega)^{N}$ and $g_{j} \in W^{2 m-m_{j}-1 / p}(\Gamma)$, and the a priori estimate

$$
\sum_{j=1}^{N}\left\|\left|u_{j}\right|\right\|_{2 m, p, \Omega} \leq C\left[\sum_{j=1}^{N}\left\|f_{j}\right\|_{0, p, \Omega}+\sum_{j=1}^{m N}\left\|\mid g_{j}\right\|_{2 m-m_{j}-1 / p, p, \Gamma}\right]
$$

holds, where the constant $C$ does not depend upon $f$, the $g_{j}$ and $\lambda$.

The proof of this result is essentially based on the explicit knowledge of the resolvent of model problems corresponding to (1.1), (1.2). We therefore start with some remarks on constant coefficient systems without lower order terms in the half-space $\mathbb{R}_{+}^{n}$.

In the following, let $A(D)=\sum_{|\alpha|=2 m} a_{\alpha} D^{\alpha}$ and $B_{j}(D)=\sum_{|\alpha|=m_{j}} b_{\alpha}^{j} D^{\alpha}$, $j=1, \ldots, m$, be partial differential operators with constant coefficients

$$
a_{\alpha}=\left(a_{\alpha}^{j k}\right)_{j, k=1, \ldots, N}, \quad b_{\alpha}^{j}=\left(b_{\alpha}^{j k}\right)_{k=1, \ldots, N}, j=1, \ldots, m N
$$

We set $B(D)=\left(B_{1}(D), \ldots, B_{m N}(D)\right)^{T}$ and denote by $I$ the $N \times N$ unit matrix. Then from an obvious modification of Definition 2.3, the differential operator $A(D)-\lambda I$ is said to be parameter-elliptic in $\mathcal{L}$ if

$$
\operatorname{det}(A(\xi)-\lambda I) \neq 0 \quad \text { for } \xi \in \mathbb{R}^{n}, \lambda \in \mathcal{L} \text { with }|\xi|+|\lambda| \neq 0
$$


The boundary problem $(A(D)-\lambda I, B(D))$ is said to be parameter-elliptic in $\mathcal{L}$ if (2.8) holds and if the boundary problem on the half-line

$$
\begin{gathered}
A\left(\xi^{\prime}, D_{n}\right) v(t)-\lambda v(t)=0 \quad \text { for } t=x_{n}>0, \\
B_{j}\left(\xi^{\prime}, D_{n}\right) v(t)=0 \quad \text { at } t=0 \text { for } j=1, \ldots, m N, \\
|v(t)| \rightarrow 0 \quad \text { as } t \rightarrow \infty
\end{gathered}
$$

has only the trivial solution for $\xi^{\prime} \in \mathbb{R}^{n-1}$ and $\lambda \in \mathcal{L}$ if $\left|\xi^{\prime}\right|+|\lambda| \neq 0$.

From a minor modification of the argument of [21, Section 3] we have the following result.

Lemma 2.5. Let the boundary problem $(A(D)-\lambda I, B(D))$ be parameter-elliptic in $\mathcal{L}$ and let $X=\left\{\left(\xi^{\prime}, t, \lambda\right)\left|\xi^{\prime} \in \mathbb{R}^{n-1}, \lambda \in \mathcal{L},\right| \xi^{\prime}|+| \lambda \mid \neq 0, t \geq 0\right\}$. Then there exists the $N \times m N$ matrix function $W\left(\xi^{\prime}, t, \lambda\right)$ defined in $X$ with the following properties:

(1) $W\left(\xi^{\prime}, t, \lambda\right)$ has derivatives of all orders with respect to $\xi^{\prime}$ and $t$ which are jointly continuous in $\xi^{\prime}, t$, and $\lambda$;

(2) $W\left(\rho \xi^{\prime}, \rho^{-1} t, \rho^{2 m} \lambda\right)=W\left(\xi^{\prime}, t, \lambda\right) \operatorname{diag}\left(\rho^{-m_{1}}, \ldots, \rho^{-m_{m N}}\right)$ for $\rho>0$;

and (3)

$$
\begin{gathered}
\left(A\left(\xi^{\prime}, D_{t}\right)-\lambda I\right) W\left(\xi^{\prime}, t, \lambda\right)=0 \quad \text { for } t>0 \\
B\left(\xi^{\prime}, D_{t}\right) W\left(\xi^{\prime}, t, \lambda\right)=I_{m N} \quad \text { at } t=0,
\end{gathered}
$$

where $I_{m N}$ denotes the $m N \times m N$ unit matrix. Furthermore, for each $\left(\xi^{\prime}, t, \lambda\right) \in X, W\left(\xi^{\prime}, t, \lambda\right)$ admits the representation

$$
W\left(\xi^{\prime}, t, \lambda\right)=\int_{\gamma_{+}\left(\xi^{\prime}, \lambda\right)} e^{i t \tau}\left(A\left(\xi^{\prime}, \tau\right)-\lambda I\right)^{-1} M\left(\xi^{\prime}, \tau, \lambda\right) d \tau,
$$

where $\gamma_{+}\left(\xi^{\prime}, \lambda\right)$ denotes a contour in $\mathbb{C}_{+}$enclosing all the zeros of $\operatorname{det}\left(A\left(\xi^{\prime}, \cdot\right)-\lambda I\right)$ having positive imaginary part, $M\left(\xi^{\prime}, \tau, \lambda\right)$ denotes an $N \times m N$ matrix function having derivatives of all orders with respect to $\xi^{\prime}$ which are jointly continuous in $\xi^{\prime}, t$, and $\lambda$, and

$$
M\left(\rho \xi^{\prime}, \rho \tau, \rho^{2 m} \lambda\right)=\rho^{2 m-1} M\left(\xi^{\prime}, \tau, \lambda\right) \operatorname{diag}\left(\rho^{-m_{1}}, \ldots, \rho^{-m_{m M}}\right) \text { for } \rho>0 .
$$

We henceforth let $F^{\prime}$ denote the Fourier transformation in $\mathbb{R}^{n-1}\left(x^{\prime} \rightarrow \xi^{\prime}\right)$. Then from [6, Section 2] we have the following result.

Lemma 2.6. Let $1<p<\infty$ and $|\lambda| \geq \lambda_{0}>0$. Then there exists a parameter dependent extension operator

$$
T(\lambda): \prod_{j=1}^{m N} W_{p}^{2 m-m_{j}-1 / p}\left(\mathbb{R}^{n-1}\right) \rightarrow \prod_{j=1}^{m N} W_{p}^{2 m-m_{j}}\left(\mathbb{R}_{+}^{n}\right)
$$

defined by $(T(\lambda) g)(x)=\left(F^{\prime-1} \Omega F^{\prime} g\right)(x)$ for $g$ in the domain of of $T(\lambda)$, where $\Omega=\Omega\left(\xi^{\prime}, x_{n}, \lambda\right)=\exp \left\{-\left(\left|\xi^{\prime}\right|+|\lambda|^{1 / 2 m}\right) x_{n}\right\}$, such that:

(1) $\gamma_{0} \circ T(\lambda)$ is the identity operator in $\prod_{j=1}^{m N} W_{p}^{2 m-m_{j}-1 / p}\left(\mathbb{R}^{n-1}\right)$, where $\gamma_{0} u$ 
denotes the trace of the function $u$ (defined in $\mathbb{R}_{+}^{n}$ ) on $\mathbb{R}^{n-1}$;

(2) if we denote by $g_{j}$ and $u_{j}$ the components of $g$ and $T(\lambda) g$, respectively, then

$$
\sum_{j=1}^{m N}\left\|\left|u_{j}\right|\right\|_{2 m, p, \mathbb{R}_{+}^{n}} \leq C \sum_{j=1}^{m N}\left\|g_{j} \mid\right\|_{2 m-m_{j}-1 / p, p, \mathbb{R}^{n-1}},
$$

where the constant $C$ does not depend upon $g$ and $\lambda$.

The key step for the proof of Theorem 2.4 is the continuity of the pseudodifferential operator given by the matrix $W$. More precisely, we have the following result.

Lemma 2.7. Let the boundary problem $(A(D)-\lambda I, B(D))$ be parameter-elliptic in $\mathcal{L}$. Let $1<p<\infty$ and $\lambda_{0}>0$. For $\lambda \in \mathcal{L}$ with $|\lambda| \geq \lambda_{0}$ let

$$
(E(\lambda) g)(x)=\left(F^{\prime-1} W\left(\cdot, x_{n}, \lambda\right) F^{\prime} g\left(\cdot, x_{n}\right)\right)\left(x^{\prime}\right), \quad g=\left(g_{1}, \ldots, g_{m N}\right)^{T} .
$$

Then the mapping

$$
E(\lambda): \prod_{j=1}^{m N} W_{p}^{2 m-m_{j}-1 / p}\left(\mathbb{R}^{n-1}\right) \longrightarrow W_{p}^{2 m}\left(\mathbb{R}_{+}^{n}\right)^{N}
$$

is continuous and if we let $g_{j}$ and $u_{j}$ denote the components of $g$ and $E(\lambda) g$, respectively, for $g$ in the domain of $E(\lambda)$, then the estimate

$$
\sum_{j=1}^{N}\left\|\left|u_{j}\right|\right\|_{2 m, p, \mathbb{R}_{+}^{n}} \leq C \sum_{j=1}^{m N}\left\|\mid g_{j}\right\|_{2 m-m_{j}-1 / p, p, \mathbb{R}^{n-1}}
$$

holds, where the constant $C>0$ does not depend upon $u$ and $\lambda$.

Proof. We observe from the definition that $E(\lambda)$ is a pseudodifferential operator in $\mathbb{R}^{n-1}$ depending upon the parameter $x_{n}$. Then in order to prove our assertion concerning $E(\lambda)$ let us put $h:=T(\lambda) g$ for $g \in \prod_{j=1}^{m N} W_{p}^{2 m-m_{j}-1 / p}\left(\mathbb{R}^{n-1}\right)$, where $T(\lambda)$ is the extension operator of Lemma 2.6. Following [21] let us fix an $\alpha$ with $|\alpha|=2 m$ and write

$$
\begin{aligned}
D^{\alpha} E(\lambda) g(x)= & D^{\alpha}\left(F^{\prime}\right)^{-1} W\left(\xi^{\prime}, x_{n}, \lambda\right)\left(F^{\prime} h\right)\left(\xi^{\prime}, 0\right) \\
= & \left(F^{\prime}\right)^{-1}\left(\xi^{\prime}\right)^{\alpha^{\prime}} D_{n}^{\alpha_{n}} W\left(\xi^{\prime}, x_{n}, \lambda\right)\left(F^{\prime} h\right)\left(\xi^{\prime}, 0\right) \\
= & -\int_{0}^{\infty} \frac{\partial}{\partial \sigma}\left[\left(F^{\prime}\right)^{-1}\left(\xi^{\prime}\right)^{\alpha^{\prime}} D_{n}^{\alpha_{n}} W\left(\xi^{\prime}, x_{n}+\sigma, \lambda\right)\left(F^{\prime} h\right)\left(\xi^{\prime}, \sigma\right)\right] d \sigma \\
= & -\int_{0}^{\infty}\left(F^{\prime}\right)^{-1}\left(\xi^{\prime}\right)^{\alpha^{\prime}} D_{n}^{\alpha_{n}+1} W\left(\xi^{\prime}, x_{n}+\sigma, \lambda\right)\left(F^{\prime} h\right)\left(\xi^{\prime}, \sigma\right) d \sigma \\
& \quad-\int_{0}^{\infty}\left(F^{\prime}\right)^{-1}\left(\xi^{\prime}\right)^{\alpha^{\prime}} D_{n}^{\alpha_{n}} W\left(\xi^{\prime}, x_{n}+\sigma, \lambda\right) D_{n}\left(F^{\prime} h\right)\left(\xi^{\prime}, \sigma\right) d \sigma \\
= & \int_{0}^{\infty} v_{1}\left(\xi^{\prime}, x_{n}, \sigma\right) d \sigma+\int_{0}^{\infty} v_{1}\left(\xi^{\prime}, x_{n}, \sigma\right) d \sigma=: u_{1}+u_{2} .(2.11)
\end{aligned}
$$


We have to estimate the $L_{p}\left(\mathbb{R}_{+}^{n}\right)^{N}$-norm of $u_{1}$ and $u_{2}$. Starting with $u_{1}$, we write

$$
\begin{gathered}
\left\|\int_{0}^{\infty} v_{1}\left(\xi^{\prime}, x_{n}, \sigma\right) d \sigma\right\|_{L_{p}\left(\mathbb{R}_{+}^{n}\right)^{N}}^{p}=\int_{0}^{\infty}\left\|\int_{0}^{\infty} v_{1}\left(\xi^{\prime}, x_{n}, \sigma\right) d \sigma\right\|_{L_{p}\left(\mathbb{R}^{n-1}\right)^{N}}^{p} d x_{n} \\
\leq \int_{0}^{\infty}\left[\int_{0}^{\infty}\left\|v_{1}\left(\xi^{\prime}, x_{n}, \sigma\right)\right\|_{L_{p}\left(\mathbb{R}^{n-1}\right)^{N}} d \sigma\right]^{p} d x_{n} .
\end{gathered}
$$

Thus we have to estimate the $L_{p}\left(\mathbb{R}^{n-1}\right)^{N}$-norm in the last expression. This norm equals

$$
\left\|\left(F^{\prime}\right)^{-1} \widetilde{W}\left(\xi^{\prime}, x_{n}+\sigma, \lambda\right)\left(F^{\prime} \widetilde{h}\right)\left(\xi^{\prime}, \sigma\right)\right\|_{L_{p}\left(\mathbb{R}^{n-1}\right)^{N}},
$$

where we have set

$$
\begin{aligned}
\widetilde{W}\left(\xi^{\prime}, x_{n}+\sigma, \lambda\right) & :=\left(\xi^{\prime}\right)^{\alpha^{\prime}} D_{n}^{\alpha_{n}+1} W\left(\xi^{\prime}, x_{n}+\sigma, \lambda\right) \Delta^{-1}(\rho), \\
\widetilde{h}\left(x^{\prime}, \sigma\right) & :=\left(F^{\prime}\right)^{-1} \Delta(\rho)\left(F^{\prime} h\right)\left(\xi^{\prime}, \sigma\right)
\end{aligned}
$$

with $\rho:=\left(\left|\xi^{\prime}\right|^{2}+|\lambda|^{1 / m}\right)^{1 / 2}$ and

$$
\Delta(\rho):=\rho^{2 m} \operatorname{diag}\left(\rho^{-m_{1}}, \ldots, \rho^{-m_{m N}}\right) .
$$

We will apply Michlin's multiplier theorem in $\mathbb{R}^{n-1}$ to $\widetilde{W}$. By definition of $W$ (see Lemma 2.5), we have

$$
\begin{aligned}
& \widetilde{W}\left(\xi^{\prime}, x_{n}+\sigma, \lambda\right) \\
& =\left(\xi^{\prime}\right)^{\alpha^{\prime}} \int_{\gamma_{+}\left(\xi^{\prime}, \lambda\right)} e^{i\left(x_{n}+\sigma\right) \tau} \tau^{\alpha_{n}+1}\left(A\left(\xi^{\prime}, \tau\right)-\lambda I\right)^{-1} M\left(\xi^{\prime}, \tau, \lambda\right) d \tau \Delta(\rho) \\
& =\rho \int_{\gamma_{+}\left(\frac{\xi^{\prime}}{\rho}, \frac{\lambda}{\rho^{2 m}}\right)} e^{i\left(x_{n}+\sigma\right) \rho \tau}\left(\frac{\xi^{\prime}}{\rho}\right)^{\alpha^{\prime}} \tau^{\alpha_{n}+1}\left[A\left(\frac{\xi^{\prime}}{\rho}, \tau\right)-\frac{\lambda}{\rho^{2 m}} I\right]^{-1} M\left(\frac{\xi^{\prime}}{\rho}, \tau, \frac{\lambda}{\rho^{2 m}}\right) d \tau,
\end{aligned}
$$

where we have used the homogeneity of the expressions concerned. Since the set of $\left(\frac{\xi^{\prime}}{\rho}, \frac{\lambda}{\rho^{2 m}}\right)$ such that $\xi^{\prime} \in \mathbb{R}^{n}, \lambda \in \mathcal{L},\left|\xi^{\prime}\right|+|\lambda| \neq 0$, is compact, the set $\mathcal{K}$ of the zeros of $\operatorname{det}\left(A\left(\frac{\xi^{\prime}}{\rho}\right)-\frac{\lambda}{\rho^{2 m}} I\right)$ with non-negative imaginary parts for these $\xi^{\prime}$ and $\lambda$ is compact. As the problem is parameter-elliptic in $\mathcal{L}$, none of the above zeros is real, i.e., $\mathcal{K} \subset \mathbb{C}_{+}$, and we can choose a contour $\gamma$ in $\mathbb{C}_{+}$surrounding $\mathcal{K}$. Then there is a constant $C_{\gamma}>0$ such that $\operatorname{Im} \tau \geq C_{\gamma}$ for all $\tau \in \gamma$. Therefore we obtain the estimate

$$
\left|\widetilde{W}\left(\xi^{\prime}, x_{n}+\sigma, \lambda\right)\right| \leq C_{0} \rho \exp \left(-C_{\gamma}\left(x_{n}+\sigma\right) \rho\right) \leq \frac{C_{0} / C_{\gamma}}{x_{n}+\sigma}
$$

with some constant $C_{0}$. Here we used the inequality $t e^{-t} \leq 1$ for $t \geq 0$.

Now let us consider $D_{\xi^{\prime}}^{\beta^{\prime}} \widetilde{W}$ instead of $\widetilde{W}$. Differentiation with respect to $\xi^{\prime}$ diminishes the homogeneity of the integrand by 1 . Therefore, we can see with the 
same considerations as before that for every $\beta^{\prime} \in \mathbb{N}_{0}^{n-1}$ there exists a constant $C_{\beta^{\prime}}$ such that the inequality

$$
\left|D_{\xi^{\prime}}^{\beta^{\prime}} \widetilde{W}\left(\xi^{\prime}, x_{n}+\sigma, \lambda\right)\right| \leq \frac{C_{\beta^{\prime}}}{x_{n}+\sigma}\left|\xi^{\prime}\right|^{-\left|\beta^{\prime}\right|} \quad\left(\xi^{\prime} \neq 0\right)
$$

holds. Applying Michlin's theorem (see e.g. [20, p. 166]) to $\widetilde{W}$, we get

$$
\left\|\left(F^{\prime}\right)^{-1} \widetilde{W}\left(\xi^{\prime}, x_{n}+\sigma, q\right)\left(F^{\prime} \widetilde{h}\right)\left(\xi^{\prime}, \sigma\right)\right\|_{L_{p}\left(\mathbb{R}^{n-1}\right)^{N}} \leq C \frac{\|\widetilde{h}(\cdot, \sigma)\|_{L_{p}\left(\mathbb{R}_{+}^{n}\right)^{m N}}}{x_{n}+\sigma},
$$

with some constant $C$. Substituting this into the last integral in (2.12), we see that

$$
\begin{aligned}
\left\|u_{1}\right\|_{L_{p}\left(\mathbb{R}_{+}^{n}\right)^{N}} & \leq C\left(\int_{0}^{\infty}\left[\int_{0}^{\infty} \frac{\|\widetilde{h}(\cdot, \sigma)\|_{L_{p}\left(\mathbb{R}^{n-1}\right)^{m N}}}{x_{n}+\sigma} d \sigma\right]^{p} d x_{n}\right)^{1 / p} \\
& \leq C_{1}\left(\int_{0}^{\infty}\left\|\widetilde{h}\left(\cdot, x_{n}\right)\right\|_{L_{p}\left(\mathbb{R}^{n-1}\right)^{m N}}^{p} d x_{n}\right)^{1 / p} \\
& =C_{1}\|\widetilde{h}\|_{L_{p}\left(\mathbb{R}_{+}^{n}\right)^{m N}}
\end{aligned}
$$

with some constant $C_{1}$. Here we used the continuity of the Hilbert transform in $L_{p}(\mathbb{R})$, applied to the function

$$
\sigma \mapsto \begin{cases}\|\widetilde{h}(\cdot, \sigma)\|_{L_{p}\left(\mathbb{R}^{n-1}\right)^{m N}} & \text { if } \sigma \geq 0, \\ 0 & \text { if } \sigma<0 .\end{cases}
$$

By definition of $\widetilde{h}$ and continuity of the extension operator $T(\lambda)$, we obtain

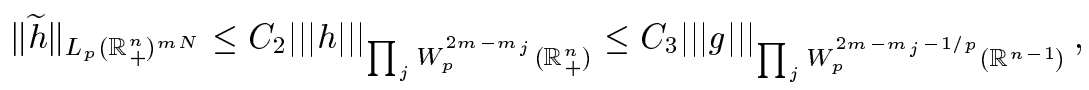

with some constants $C_{2}, C_{3}$. Analogously we get the same estimate for $u_{2}$ in (2.11) and for $|\lambda|\|E(\lambda) g\|_{L_{p}\left(\mathbb{R}_{+}^{n}\right)^{N}}$. Because

$$
\begin{aligned}
\||E(\lambda) g|\|_{W_{p}^{2 m}\left(\mathbb{R}_{+}^{n}\right)^{N}} \leq & C\left(\sum_{|\alpha|=2 m}\left\|D^{\alpha} E(\lambda) g\right\|_{L_{p}\left(\mathbb{R}_{+}^{n}\right)^{N}}\right. \\
& \left.+|\lambda|\|E(\lambda) g\|_{L_{p}\left(\mathbb{R}_{+}^{n}\right)^{N}}\right),
\end{aligned}
$$

this finishes the proof of the lemma.

Proof of Theorem 2.4. As the proof follows a standard approach in elliptic theory using localization (freezing the coefficients) and can be established to a large extent in a similar way as the corresponding proof in [6], we only concentrate on the model problems. The general result for operators with variable coefficients in a bounded domain then can be obtained exactly in the same way as in [6]. In the following, all Sobolev spaces are endowed with the parameter-dependent norms, and continuity of a family of parameter-dependent operators has to be understood in the sense that these operators are bounded, and their norm with respect to the 
parameter-dependent Sobolev spaces can be estimated by a constant independent of the parameter.

(i) We first assume $\omega(x) \equiv I$ and localize the operator in the neighbourhood of a point in $\Omega \backslash \bigcup_{r=1}^{N_{0}} \Gamma_{r}$ which does not intersect with $\Gamma_{r}$ for $r=0, \ldots, N_{0}$, where $\Gamma_{0}=\Gamma$. We obtain as a model problem an operator of the form $A(D)-\lambda I$ with constant coefficients and without lower-order terms which acts in the whole space $\mathbb{R}^{n}$ and is parameter-elliptic in $\mathcal{L}$. It is easily seen, using Michlin's theorem, that this operator, acting from $W_{p}^{2 m}\left(\mathbb{R}^{n}\right)^{N}$ into $L_{p}\left(\mathbb{R}^{n}\right)^{N}$, is invertible for every $\lambda \in \mathcal{L} \backslash\{0\}$ with inverse $R(\lambda):=F^{-1}(A(\xi)-\lambda I)^{-1} F$. Moreover, the family $\left\{R(\lambda): \lambda \in \mathcal{L},|\lambda| \geq \lambda_{0}\right\}$ is continuous for every $\lambda_{0}$ in the sense described above. This is the exact analog of the result in [6].

(ii) Now let us localize in a neighbourhood of a point $x_{0} \in \Gamma$, still assuming $\omega(x) \equiv I$. Freezing the coefficients at $x_{0}$ and taking the principal parts of the operators, we obtain a model problem in $\mathbb{R}_{+}^{n}$ of the form

$$
\begin{aligned}
(A(D)-\lambda I) u=f & & \text { in } \mathbb{R}_{+}^{n} \\
B(D) u=g & & \text { on } \mathbb{R}^{n-1} .
\end{aligned}
$$

For $f \in L_{p}\left(\mathbb{R}_{+}^{n}\right)^{N}$ and $g \in \prod_{j=1}^{m N} W_{p}^{2 m-m_{j}-1 / p}(\Gamma)$, a solution $u \in W_{p}^{2 m}\left(\mathbb{R}_{+}^{n}\right)$ is explicitly given by

$$
u:=R_{+} R(\lambda) E_{0} f+E(\lambda)\left[g-B(D) R_{+} R(\lambda) E_{0} f\right],
$$

where $E_{0}: L_{p}\left(\mathbb{R}_{+}^{n}\right)^{N} \rightarrow L_{p}\left(\mathbb{R}^{n}\right)^{N}$ denotes extension by $0, R_{+}: W_{p}^{2 m}\left(\mathbb{R}^{n}\right)^{N} \rightarrow$ $W_{p}^{2 m}\left(\mathbb{R}_{+}^{n}\right)^{N}$ denotes restriction onto $\mathbb{R}_{+}^{n}$ and where $R(\lambda)$ and $E(\lambda)$ are defined in part (i) and Lemma 2.7, respectively.

To prove the a priori estimate for the model problem (2.13), (2.14), we use the continuity of the operator

$$
R_{+} R(\lambda) E_{0}: L_{p}\left(\mathbb{R}_{+}^{n}\right)^{N} \rightarrow W_{p}^{2 m}\left(\mathbb{R}_{+}^{n}\right)^{N}
$$

(see part (i)), of the boundary operator

$$
B(D): W_{p}^{2 m}\left(\mathbb{R}_{+}^{n}\right)^{N} \rightarrow \prod_{j=1}^{m N} W_{p}^{2 m-m_{j}-1 / p}\left(\mathbb{R}^{n-1}\right)
$$

and of $E(\lambda)$ (see Lemma 2.7). Here the continuity of $B(D)$ (with respect to the parameter-dependent norms) was shown in [6, Section 2]. From this we see that for $u$ defined by (2.15) the a priori estimate

$$
\begin{array}{r}
\sum_{j=1}^{N}\|\| u_{j} \|\left.\right|_{2 m, p, \mathbb{R}_{+}^{n}} \leq C\left[\sum_{j=1}^{N}\left\|f_{j}\right\|_{0, p, \mathbb{R}_{+}^{n}}+\sum_{j=1}^{m N}\left\|g_{j} \mid\right\|_{2 m-m_{j}-1 / p, p, \mathbb{R}^{n-1}}\right] \\
\left(\lambda \in \mathcal{L},|\lambda| \geq \lambda_{0}\right)
\end{array}
$$

holds, where the constant $C$ does not depend upon $f, g$, and $\lambda$. That this solution is the unique solution in $W_{p}^{2 m}\left(\mathbb{R}_{+}^{n}\right)^{N}$ of (2.13), (2.14) is an immediate consequence of Lemma 2.5 and our parameter-ellipticity assumption. 
(iii) In the third step we consider the model problem obtained after localization at a point $x_{0} \in \Gamma_{r}$ for some $r \in\left\{1, \ldots, N_{0}\right\}$, still assuming $\omega(x) \equiv I$. Now we get a transmission problem of the form

$$
\begin{aligned}
\left(A^{+}(D)-\lambda I\right) u^{+} & =f^{+} \quad \text { in } \mathbb{R}_{+}^{n}, \\
\left(A^{-}(D)-\lambda I\right) u^{-} & =f^{-} \quad \text { in } \mathbb{R}_{-}^{n}, \\
D_{n}^{\ell} u^{+}-D_{n}^{\ell} u^{-} & =0 \quad(\ell=0, \ldots, 2 m-1) \quad \text { on } \mathbb{R}^{n-1} .
\end{aligned}
$$

We set

$$
\begin{aligned}
u(x):= & \left(\begin{array}{c}
u^{+}(x) \\
u^{-}\left(x^{\prime},-x_{n}\right)
\end{array}\right), f(x):=\left(\begin{array}{c}
f^{+}(x) \\
f^{-}\left(x^{\prime},-x_{n}\right)
\end{array}\right), \\
& A(D):=\left(\begin{array}{cc}
A^{+}(D) & 0 \\
0 & A^{-}\left(D^{\prime},-D_{n}\right)
\end{array}\right)
\end{aligned}
$$

and define the boundary operator $B(D)$ as the block matrix

$$
B(D)=\left(D_{n}^{\ell} I,(-1)^{\ell+1} D_{n}^{\ell} I\right)_{\ell=0, \ldots, 2 m-1}
$$

of dimension $2 m N \times 2 N$. We obtain a boundary value problem in $\mathbb{R}_{+}^{n}$ which satisfies the condition of parameter-ellipticity by Definition 2.3. Therefore, we can apply the results of part (ii) and get unique solvability of (2.16)-(2.18) in the analogous Sobolev space setting and the a priori estimate

$$
\begin{aligned}
\sum_{j=1}^{N}\left\|u_{j}^{+}\right\| \|_{2 m, p, \mathbb{R}_{+}^{n}} & +\sum_{j=1}^{N}\|\| u_{j}^{-} \|\left.\right|_{2 m, p, \mathbb{R}_{-}^{n}} \leq \\
& \leq C\left(\sum_{j=1}^{N}\left\|f_{j}^{+}\right\|_{0, p, \mathbb{R}_{+}^{n}}+\sum_{j=1}^{N}\left\|f_{j}^{-}\right\|_{0, p, \mathbb{R}_{-}^{n}}\right)
\end{aligned}
$$

for $\lambda \in \mathcal{L},|\lambda| \geq \lambda_{0}>0$, where the constant $C$ does not depend upon the $f^{ \pm}$ and $\lambda$.

(iv) As mentioned above, the desired result for $\omega \equiv I$ now follows by localization, using a partition of unity, in a standard way. Note that in steps (i)-(iii) we did not assume continuity of the coefficient $a_{\alpha}$ for $|\alpha|=2 m$ in $\bar{\Omega}$, but only continuity in each $\Omega_{r}, 0 \leq r \leq N_{0}$, with continuous extension to $\bar{\Omega}_{r}$.

Finally, to consider the case of a general matrix function $\omega$ satisfying Assumption 2.1, we multiply both sides of the equality

$$
(A(x, D)-\lambda \omega(x)) u(x)=f(x) \quad \text { in } \Omega
$$

by $\omega(x)^{-1}$ and obtain the boundary problem for

$$
\left(\omega(x)^{-1} A(x, D)-\lambda I, B(D)\right),
$$

where the right-hand side $f$ is replaced by $\omega^{-1} f$. Hence it follows that we can apply our previous results and obtain the a priori estimate for the boundary problem (2.19). Moreover, as the entries of $\omega^{-1}$ are in $L_{\infty}(\Omega)$, we have

$$
\left\|\omega^{-1} f\right\|_{L_{p}(\Omega)^{N}} \leq C\|f\|_{L_{p}(\Omega)^{N}},
$$

where the constant $C$ depends only upon $\omega$, and therefore the a priori estimate of Theorem 2.4 holds for general $\omega$. 


\section{Some spectral consequences}

For the remainder of this paper it will be supposed that the boundary problem $(1.1),(1.2)$ is elliptic with parameter in some sector $\mathcal{L}$. Then as a consequence of Theorem 2.4 we are now in a position to derive some information concerning the spectral properties of the problem (1.1), (1.2). Accordingly, for $1<p<\infty$ let $A_{p}$ denote the operator in $L_{p}(\Omega)^{N}$ that acts as $A(x, D)$ and has the domain

$$
D\left(A_{p}\right)=\left\{u \in W_{p}^{2 m}(\Omega)^{N} \mid B_{j}(x, D) u(x)=0 \text { on } \Gamma \text { for } j=1, \ldots, m N\right\},
$$

and let $V_{p}$ denote the operator of multiplication induced in $L_{p}(\Omega)^{N}$ by $\omega(x)$ (see the third paragraph of Section 1). Then it follows from Theorem 2.4 that $V_{p}^{-1} A_{p}$ is a densely defined closed operator in $L_{p}(\Omega)^{N}$ with non-empty resolvent set and compact resolvent and that for $\lambda \in \mathcal{L}$ with $|\lambda| \geq \lambda_{0}(p)$, the estimate

$$
\left\|\left(V_{p}^{-1} A_{p}-\lambda I\right)^{-1} f\right\|_{W_{p}^{2 m}(\Omega)^{N}} \leq C\|f\|_{L_{p}(\Omega)^{N}}
$$

holds for every $f \in L_{p}(\Omega)^{N}$, where the constant $C$ does not depend upon $f$ and $\lambda$. Thus we have shown that $V_{p}^{-1} A_{p}$ has a discrete spectrum, while arguments somewhat similar to those used in [2] show that the eigenvalues and principal vectors of $V_{p}^{-1} A_{p}$ do not depend upon $p$. Because of this we will be mainly concerned with the operator $V^{-1} A$, where $V=V_{2}$ and $A=A_{2}$. It is important to observe from (3.1) and [2] that if we let $R(\lambda)$ denote the resolvent of $V^{-1} A$, then $R(\lambda)$ is a compact operator in $L_{2}(\Omega)^{N}$ of class $\mathcal{C}_{\frac{n}{2 m}+\varepsilon}$ for every $\varepsilon>0$.

The following four results, which were presented in [6] for the scalar problem considered there, carry over directly to the matrix problem under consideration here (see [2], [4, pp. 116-117], [8, p. 1115]). Here $L_{2, \omega}(\Omega)^{N}$ denotes the Hilbert space with inner product $\left(\operatorname{diag}\left(\left|\omega_{1}(\cdot)\right|, \ldots,\left|\omega_{N}(\cdot)\right| \cdot, \cdot\right)\right.$, where $(\cdot, \cdot)$ denotes the inner product in $L_{2}(\Omega)^{N}$.

Theorem 3.1. Suppose that the boundary problem (1.1), (1.2) is elliptic with parameter along some rays $\mathcal{L}_{j}, j=1, \ldots, q$, and the angle between any two adjacent rays is not greater than $2 m \pi / n$. Then $V^{-1} A$ has an infinite number of eigenvalues and the set of principal vectors of $V^{-1} A$ is complete in each of the Hilbert spaces $L_{2}(\Omega)^{N}$ and $L_{2, w}(\Omega)^{N}$.

Assuming that the spectrum of $V^{-1} A$ is not empty, let $\left\{\lambda_{j}\right\}_{j \geq 1}$ denote the sequence of all eigenvalues of $V^{-1} A$ arranged so that $\left|\lambda_{1}\right| \leq\left|\lambda_{2}\right| \leq \ldots$, where each eigenvalue is repeated according to its multiplicity. Let $\left\{u_{j}\right\}_{j \geq 1}$ denote the system of principal vectors of $V^{-1} A$ arranged so the $u_{j}$ belongs to principal subspace of $V^{-1} A$ corresponding to the eigenvalue $\lambda_{j}$. Then under the assumptions of Theorem 3.1 the $u_{j}$ form an infinite complete minimal system in $L_{2}(\Omega)^{N}$ and there exists a system $\left\{w_{j}\right\}_{1}^{\infty}$ which is biorthogonal to $\left\{u_{j}\right\}_{1}^{\infty}$. To each $f \in L_{2}(\Omega)^{N}$ we can then associate its formal Fourier series with respect to $\left\{u_{j}\right\}_{1}^{\infty}$,

$$
f \sim \sum_{j=1}^{\infty} c_{j} u_{j}, \text { where } c_{j}=\left(f, w_{j}\right)
$$


Theorem 3.2. Let $f \in L_{2}(\Omega)^{N}$. Then under the assumptions of Theorem 3.1 the series (3.2) admits the summability to $f$ in both $L_{2}(\Omega)^{N}$ and $L_{2, \omega}(\Omega)^{N}$ by the Abel-Lidskii method of order $\frac{n}{2 m}+\varepsilon$ if $\varepsilon>0$ is sufficiently small.

For information regarding Abel-Lidskii summability we refer the reader to [4, p. 116], [6, Section 3]. Now let $\mathcal{L}(\theta)=\overline{\{\lambda \mid \arg \lambda=\theta\}}$.

Theorem 3.3. Suppose that the boundary problem (1.1), (1.2) is elliptic with parameter for each of the rays $\mathcal{L}=\mathcal{L}\left(\theta_{1}\right)$ and $\mathcal{L}=\mathcal{L}\left(\theta_{2}\right)$, where $0<\theta_{2}-\theta_{1}<$ $\min \{2 m \pi / n, 2 \pi\}$, but not elliptic with parameter for the ray $\mathcal{L}=\mathcal{L}\left(\theta_{0}\right)$, where $\theta_{1}<\theta_{0}<\theta_{2}$. Then the sector $\left\{\lambda \mid \theta_{1}<\arg \lambda<\theta_{2}\right\}$ contains infinitely many eigenvalues of $V^{-1} A$.

Theorem 3.4. Suppose that for some $\varepsilon>0$ the boundary problem (1.1), (1.2) is elliptic with parameter for each of the rays $\mathcal{L}(\theta)$ for $\theta_{0}-\varepsilon<\theta<\theta_{0}$ and $\theta_{0}<\theta<\theta_{0}+\varepsilon$, but is not elliptic with parameter for the ray $\mathcal{L}\left(\theta_{0}\right)$. Then any angular neighbourhood of $\mathcal{L}\left(\theta_{0}\right)$ contains infinitely many eigenvalues of $V^{-1} A$.

\section{Eigenvalue asymptotics}

Throughout this section it will be supposed that the boundary problem (1.1), (1.2) is weakly smooth and elliptic with parameter in $\mathcal{L}$. Then as a consequence of the foregoing results we are now in a position to derive information concerning the asymptotic behaviour of the eigenvalues of $V^{-1} A$. Furthermore, since we have already shown that $V^{-1} A$ has a discrete spectrum, there is no loss of generality in supposing henceforth that 0 is in the resolvent set of $V^{-1} A$.

We already know from our discussion of Section 1 and Proposition A.6 that the analogue of Theorem 2.4 also holds for the boundary problem (1.5), (1.6). Hence if for $1<p<\infty$ we let $A_{p}^{\prime}$ denote the operator in $L_{p}(\Omega)^{N}$ which acts as $A^{\prime}(x, D)$ and has the domain

$$
D\left(A_{p}^{\prime}\right)=\left\{u \in W_{p}^{2 m}(\Omega)^{N} \mid B_{j}^{\prime}(x, D) u(x)=0 \text { on } \Gamma \text { for } j=1, \ldots, m N\right\},
$$

then it follows that $V_{p}^{-1} A_{p}^{\prime}$ is a densely defined closed operator in $L_{p}(\Omega)^{N}$ with non-empty resolvent set and compact resolvent and that the estimate (3.1) also holds when $A_{p}$ is replaced by $A_{p}^{\prime}$ and $\lambda$ by $\bar{\lambda}$. Observing that $A_{p}$ and $A_{p}^{\prime}$ are closed densely defined operators in $L_{p}(\Omega)^{N}$, it is also not difficult to show that $A_{p}^{*}=A_{p^{\prime}}^{\prime}$, where $A_{p}^{*}$ denotes the adjoint of $A_{p}$ and $1 / p+1 / p^{\prime}=1$. Indeed, by appeal to the Green formula (see the proof of Proposition A.6), it is readily seen that $A_{p^{\prime}}^{\prime} \subset A_{p}^{*}$, while Theorem 2.4 and its analogue for the boundary problem (1.5), (1.6) assure us that this inclusion cannot be proper. We shall now use these facts to arrive at the required results.

For $0<\theta \leq \pi$ let $\mathcal{L}_{\theta}$ denote the closed sector in the $\lambda$-plane with vertex at the origin defined by the inequalities $\theta \leq|\arg \lambda| \leq \pi$. Then we have the following result concerning the resolvent $R(\lambda)$ of $V^{-1} A$. 
Theorem 4.1. Suppose that the boundary problem (1.1), (1.2) is weakly smooth and elliptic with parameter in $\mathcal{L}_{\theta}$. Let $q$ be an even integer satisfying $q>n / 2 m$. Then $R(\lambda)^{q}$ is an integral operator of trace class. Furthermore, if we let $\operatorname{tr} R(\lambda)^{q}$ denote the trace of $R(\lambda)^{q}$, then

$$
\operatorname{tr} R(\lambda)^{q}=c_{q}(-\lambda)^{\frac{n}{2 m}-q}+o\left(|\lambda| \frac{n}{2 m}-q\right) \text { as }|\lambda| \rightarrow \infty, \lambda \in \mathcal{L}_{\theta},
$$

uniformly in $\lambda$, where

$$
c_{q}=\int_{\Omega} c_{q}(x) d x \text { and } c_{q}(x)=\frac{1}{(2 \pi)^{n}} \int_{\mathbb{R}^{n}} \operatorname{tr}\left(\omega(x)^{-1} \stackrel{\circ}{A}(x, \xi)+I\right)^{-q} d \xi,
$$

and the holomorphic function $(-\lambda) \cdots$ is defined outside of $\overline{\mathbb{R}_{+}}$and is equal $|\lambda| \cdots$ when $\lambda \in \mathbb{R}_{\text {_. }}$.

Before beginning the proof let us observe for later use that

$$
\operatorname{tr}\left(w(x)^{-1} \stackrel{\circ}{A}(x, \xi)+I\right)^{-q}=\sum_{j=1}^{N}\left(\lambda_{j}(x, \xi)+1\right)^{-q},
$$

where the $\lambda_{j}(x, \xi)$ denote the eigenvalues of $\omega(x)^{-1} \stackrel{\circ}{A}(x, \xi)$. Furthermore, with the aid of Rouché's theorem, we can also show that the $\lambda_{j}(x, \xi)$ are continuous in $\overline{\Omega_{r}} \times \mathbb{R}^{n} \backslash\{0\}$ for $r=0, \ldots, N_{0}$ in the sense described in [14, p. 108].

Proof. Since the proof is essentially a repetition of the proofs of [6, Theorems 5.1, 8.8], we shall content ourselves with merely sketching an outline. We shall also henceforth suppose that $\lambda \in \mathcal{L}_{\theta}$ with $|\lambda|$ sufficiently large.

In light of the properties of $R(\lambda)$ cited in Section 3, it follows from [6, Section 4], [10, p. 92] that $R(\lambda)^{q}$ is a compact operator in $L_{2}(\Omega)^{N}$ of trace class. Next observing that $R(\lambda)^{*}=V R^{\prime}(\bar{\lambda}) V^{-1}$, where ${ }^{*}$ denotes adjoint and $R^{\prime}(\lambda)$ denotes the resolvent of $V^{-1} A_{2}^{\prime}$, we can write $R(\lambda)^{q}=R(\lambda)^{k}\left(R^{\prime}(\bar{\lambda})^{k} V^{-1}\right)^{*} V$, where $k=q / 2$. Let $\left\{p_{j}\right\}_{1}^{k}$ be numbers satisfying $2=p_{1}<p_{2}<\ldots<p_{k}<\infty$, $\frac{n}{2 m}\left(\frac{1}{p_{j}}-\frac{1}{p_{j+1}}\right)<1$ for $j=1, \ldots, k-1, p_{k}>n / 2 m$. Then bearing in mind that (3.1) implies that the mapping $R(\lambda): L_{p_{j}}(\Omega)^{N} \rightarrow W_{p_{j}}^{2 m}(\Omega)^{N}$ is continuous, while the Sobolev embedding theorem (see [6, Section 2]) assures us that $W_{p_{j}}^{2 m} \hookrightarrow$ $L_{p_{j+1}}(\Omega)^{N}$, where $p_{k+1}=\infty$ and $L_{\infty}(\Omega)^{N}$ is equipped with its usual norm (see [1, p. 46]), we can consider $R(\lambda)^{k}: L_{2}(\Omega)^{N} \rightarrow L_{\infty}(\Omega)^{N}$ as a composition of the mappings

$$
L_{2}(\Omega)^{N} \stackrel{R(\lambda)}{\longrightarrow} L_{p_{2}}(\Omega)^{N} \stackrel{R(\lambda)}{\longrightarrow} \ldots \stackrel{R(\lambda)}{\longrightarrow} L_{p_{k}}(\Omega)^{N} \stackrel{R(\lambda)}{\longrightarrow} L_{\infty}(\Omega)^{N} .
$$

In light of this fact we can now appeal to (3.1), the Sobolev embedding theorems (see [1, p. 97], [6, Section 2]), the properties of Hilbert-Schmidt operators cited in [10, pp. 106-112] and argue as in [6] to deduce that $R(\lambda)^{k}$ is a Hilbert-Schmidt operator whose kernel $K_{1}(x, y, \lambda)$ satisfies

$\int_{\Omega}\left|K_{1}(x, y, \lambda)\right|^{2} d y \leq c|\lambda|^{\frac{n}{2 m}-q}, \int_{\Omega}\left|K_{1}(x, y, \lambda)-K_{1}(\tilde{x}, y, \lambda)\right|^{2} d y \leq c|x-\tilde{x}|^{\gamma}$ 
for $x, \tilde{x} \in \bar{\Omega}$ and some $\gamma>0$, where the constant $c$ does not depend upon $x$, $\tilde{x}$, and $\lambda$, and for any $N \times N$ matrix $\mathcal{A},|\mathcal{A}|^{2}=\operatorname{tr} \mathcal{A}^{*} \mathcal{A}$. Since a similar result holds for $R(\bar{\lambda})^{k} V^{-1}$, arguments similar to those used in [6] show that for $\lambda \in \mathcal{L}_{\theta}$ with $|\lambda|$ sufficiently large, $R(\lambda)^{q}$ is an integral operator with kernel $K_{\omega}(x, y, \lambda)=$ $K(x, y, \lambda) w(y)$, where $K(x, y, \lambda)$ is continuous in $\bar{\Omega} \times \bar{\Omega}$ and

$$
|K(x, y, \lambda)| \leq c|\lambda|^{\frac{n}{2 m}-q},
$$

where the constant $c$ does not depend upon $x, y, \lambda$. Finally, using this result and a localization argument as in [6] we can show that

$$
\int_{\Omega}\left|K_{\omega}(x, x, \lambda)-\frac{1}{(2 \pi)^{n}} \int_{\mathbb{R}^{n}}\left(\omega(x)^{-1} \stackrel{\circ}{\AA}(x, \xi)-\lambda I\right)^{-q} d \xi\right| d x=o\left(|\lambda|^{\frac{n}{2 m}-q}\right)
$$

as $|\lambda| \rightarrow \infty, \lambda \in \mathcal{L}_{\theta}$, uniformly in $\mathcal{L}_{\theta}$, and all the assertions now follow.

Referring again to (4.2) let us next put $c_{q}=b_{n / 2 m, q} d_{q}$, where $b_{t, q}=t B(t, q-t)$, and $B(t, s)$ is the Beta function. For later use let us observe from Proposition A.1 that for the case where $\AA(x, \xi)$ is positive definite in $\bar{\Omega} \times \mathbb{R}^{n} \backslash\{0\}$ and the components of $\omega(x)$ are all positive in $\overline{\Omega_{r}}$ for $r=0, \ldots, N_{0}$, the $\lambda_{j}(x, \xi)$ of (4.3) are all positive. Hence it follows from (4.2), (4.3), [5, Eq. (1.2.9)], and the fact that each $\lambda_{j}(x, \xi)$ is continuous in $\overline{\Omega_{r}} \times \mathbb{R}^{n} \backslash\{0\}$ for $r=0, \ldots, N_{0}$ (see [14, p. 109]) that for this case

$$
d_{q}=d=\frac{1}{(2 \pi)^{n} n} \int_{\Omega} d x \sum_{j=1}^{N} \int_{|\xi|=1} \lambda_{j}(x, \xi)^{-n / 2 m} d S_{\xi}
$$

We now come to our first result concerning the eigenvalue asymptotics. Accordingly, let us introduce the counting function $N(t)=\max \left\{j|| \lambda_{j} \mid \leq t\right\}$. Now observe that if the boundary problem (1.1), (1.2) is elliptic with parameter in $\mathcal{L}$ and the constant $\rho(|\rho|=1)$ is suitably chosen, then the boundary problem $(1.1)^{\prime}$, (1.2) is elliptic with parameter in $\mathcal{L}_{\theta}$, where (1.1)' is obtained from (1.1) by replacing $A(x, D)$ by $\rho A(x, D)$ and $\lambda$ by $\rho \lambda$. Furthermore, $N(t)$ is invariant under this rotation. Thus it follows that when obtaining estimates for $N(t)$, there is no loss of generality in supposing that the sector $\mathcal{L}$ of Definition 2.3 coincides with the sector $\mathcal{L}_{\theta}$ of Theorem 4.1 and that the sector $\mathcal{L}_{\theta}$ is contained in the resolvent set of $V^{-1} A$. With this in mind, we have next

Theorem 4.2. Suppose that the boundary problem (1.1), (1.2) is weakly smooth and elliptic with parameter in $\mathcal{L}_{\theta}$. Then $\varlimsup_{t \rightarrow \infty} N(t) t^{-n / 2 m}<\infty$, while if $d_{q} \neq 0$, then $\varliminf_{t \rightarrow \infty} N(t) t^{-n / 2 m}>0$.

Proof. We have shown in the proof of Theorem 4.1 that for $\lambda \in \mathcal{L}_{\theta}$ with $|\lambda|$ sufficiently large, $R(\lambda)^{k}$ is a Hilbert-Schmidt operator whose kernel $K_{1}(x, y, \lambda)$ satisfies $\int_{\Omega \times \Omega}\left|K_{1}(x, y, \lambda)\right|^{2} d x d y \leq c|\lambda|^{\frac{n}{2 m}-q}$, where the constant $c$ does not depend upon $\lambda$. Thus it follows that

$$
S_{q}(\mu)=\int_{0}^{\infty}(t+\mu)^{-q} d N(t) \leq \sum_{j \geq 1} \frac{1}{\left|\lambda_{j}+\mu\right|^{q}} \leq c \mu^{\frac{n}{2 m}-q}
$$


for all $\mu$ sufficiently large, and hence we conclude that

$$
\varlimsup_{\mu \rightarrow \infty} S_{q}(\mu) \mu^{q-\frac{n}{2 m}} \leq c .
$$

The first assertion of the theorem is an immediate consequence of this last inequality (see [5]). Furthermore, it is shown in [5] that if $d_{q} \neq 0$, then (4.5) implies that

$$
\varliminf_{\mu \rightarrow \infty} S_{q}(\mu) \mu^{q-\frac{n}{2 m}} \geq c_{0}>0
$$

and as pointed out in [5], (4.5) and (4.6) imply the second assertion of the theorem. $\square$

From Theorem 4.2 we have the following corollary.

Theorem 4.3. If $d_{q} \neq 0$, then $N(t) \asymp t^{n / 2 m}$ as $t \rightarrow \infty$.

This means that the ratio $N(t) / t^{n / 2 m}$ lies between positive numbers for large $t$.

Theorem 4.1 can also be used to sharpen this last result for the case where $\stackrel{\circ}{A}(x, \xi)$ is positive definite in $\bar{\Omega} \times \mathbb{R}^{n} \backslash\{0\}$ and the components of $\omega(x)$ all have the same sign in $\overline{\Omega_{r}}$ for $r=0, \ldots, N_{0}$, with the sign being the same for all $r$. Fixing our attention upon this case, let us observe from the proof of Proposition A. 1 that if the sign is positive and $\theta \neq 2 k \pi$ for $k \in \mathbb{Z}$, then Conditions 1 and 3 of Definition 2.3 are satisfied for $\mathcal{L}=\mathcal{L}(\theta)$, and hence in order that the boundary problem (1.1), (1.2) be elliptic with parameter in $\mathcal{L}(\theta)$, we only require that the Condition 2 be satisfied. Note from Proposition A.2 that this will always be the case if the boundary conditions (1.2) are of Dirichlet type. On the other hand, if $\theta=2 k \pi$ for some $k \in \mathbb{Z}$, then the boundary problem (1.1), (1.2) cannot be elliptic with parameter in $\mathcal{L}(\theta)$. Let us now suppose that the sign is positive (the case where the sign is negative can be dealt with in a similar fashion). Then we observe from (4.4) that $d>0$, and hence it follows from Theorem 4.3 that $V^{-1} A$ has an infinite number of eigenvalues. Furthermore, we observe from Theorem 3.4 that for any $\varepsilon$ satisfying $0<\varepsilon<\pi / 2$, there are infinitely many eigenvalues of $V^{-1} A$ lying in the sector $|\arg \lambda|<\varepsilon$, while there are at most only a finite number of eigenvalues lying in the sector $|\arg \lambda-\pi| \leq \pi-\varepsilon$.

Theorem 4.4. Suppose that $\stackrel{\circ}{A}(x, \xi)$ is positive definite in $\bar{\Omega} \times \mathbb{R}^{n} \backslash\{0\}$ and that the components of $\omega(x)$ are all positive in $\overline{\Omega_{r}}$ for $r=0, \ldots, N_{0}$. Suppose also that the boundary problem (1.1), (1.2) is weakly smooth and that Condition 2 of Definition 2.3 holds for every ray $\mathcal{L}=\mathcal{L}(\theta)$ for which $\theta \neq 2 k \pi, k \in \mathbb{Z}$. Then

$$
N(t)=\kappa t^{n / 2 m}+o\left(t^{n / 2 m}\right) \text { as } t \rightarrow \infty,
$$

where

$$
\kappa=\frac{1}{(2 \pi)^{n}} \int_{\Omega} d x \sum_{j=1}^{N} \int_{\lambda_{j}(x, \xi)<1} d \xi
$$


Proof. It follows from Theorem 4.1 and (4.4) that

$$
\sum_{j=1}^{\infty} \frac{1}{\left(\lambda_{j}+t\right)^{q}}=b_{n / 2 m, q} d t^{\frac{n}{2 m}-q}+o\left(t^{\frac{n}{2 m}-q}\right) \text { as } t \rightarrow \infty,
$$

and hence we can appeal to Theorem 4.3 and argue in a manner somewhat similar to the way we argued in the proof of [6, Theorem 6.3] to deduce that

$$
\sum_{j=1}^{\infty} \frac{1}{\left(\left|\lambda_{j}\right|+t\right)^{q}}=b_{n / 2 m, q} d t^{\frac{n}{2 m}-q}+o\left(t^{\frac{n}{2 m}-q}\right) \text { as } t \rightarrow \infty
$$

The assertion of the theorem now follows from the Hardy-Littlewood Tauberian theorem (see [5] and [19, p. 110]).

Our goal now is to obtain the analogue of Theorem 4.4 when for at least one $r, 0 \leq r \leq N_{0}$, the components of $\omega(x)$ are not all of the same sign in $\Omega_{r}$. Since Theorem 4.1 is no longer adequate to deal with this case, some preparation is required. To this end we firstly require the following extension of Theorem 4.1, the proof of which is given in [6, Section 6].

Theorem 4.5. Suppose that $q>n / 2 m$ and $q$ is not an even integer. Let $s$ denote the odd integer satisfying $|s-q|<1$. Then the asymptotic formula (4.1) holds with $c_{q}=\frac{b_{n / 2 m, q}}{b_{n / 2 m, s+1}} c_{s+1}$.

Suppose next that the boundary problem (1.1), (1.2) is weakly smooth and elliptic with parameter in two closed sectors $\mathcal{L}=\mathcal{L}^{(1)}$ and $\mathcal{L}=\mathcal{L}^{(2)}$ which intersect only at the origin. Let $\Lambda^{+}$and $\Lambda^{-}$denote the open sectors complementary to $\mathcal{L}^{(1)} \cup \mathcal{L}^{(2)}$. Without loss of generality we may assume that all the eigenvalues of $V^{-1} A$ lie in $\Lambda^{+}$and $\Lambda^{-}$and that $\overline{\mathbb{R}_{+}}$is the bisectrix of $\Lambda^{+}$. Let us also suppose that the eigenvalues of $\omega(x)^{-1} \stackrel{\circ}{A}(x, \xi)$ are all real-valued for $x \in \overline{\Omega_{r}} \times \mathbb{R}^{n} \backslash\{0\}$, $r=0, \ldots, N_{0}$, and that for at least one $r$, there is a $j, 1 \leq j \leq N$, such that $\lambda_{j}(x, \xi) \in \Lambda^{+}$(resp. $\left.\lambda_{j}(x, \xi) \in \Lambda^{-}\right)$at some point $(x, \xi) \in \overline{\Omega_{r}} \times \mathbb{R}^{n} \backslash\{0\}$. Fixing our attention upon the case $\lambda_{j}(x, \xi) \in \Lambda^{+}$, let us denote by $n_{r}^{+}(x, \xi)$ the number of such eigenvalues. Now suppose that for at least one $j, \lambda_{j}\left(x^{0}, \xi^{0}\right) \in \Lambda^{+}$ for $\left(x^{0}, \xi_{0}\right) \in \overline{\Omega_{r}} \times S^{n-1}$, where $S^{n-1}=\left\{\xi \in \mathbb{R}^{n}|| \xi \mid=1\right\}$. Then it follows from Rouché's theorem that there exists the relatively open subset $U$ of $\overline{\Omega_{r}} \times S^{n-1}$ containing $\left(x^{0}, \xi^{0}\right)$ such that $n_{r}^{+}(x, \xi)$ is constant in $U$. Hence, since $\overline{\Omega_{r}} \times S^{n-1}$ is connected, we conclude that $n_{r}^{+}(x, \xi)$ is constant in $\overline{\Omega_{r}} \times S^{n-1}$. Bearing in mind that the eigenvalues of $\omega(x)^{-1} \stackrel{\circ}{A}(x, \xi)$ are homogeneous with respect to $\xi$ of degree $2 m$, it follows that there exists the integer $n_{r}^{+}, 1 \leq n_{r}^{+} \leq N$, such that $\omega(x)^{-1} \stackrel{\circ}{A}(x, \xi)$ has precisely $n_{r}^{+}$eigenvalues lying in $\Lambda^{+}$for $(x, \xi) \in \overline{\Omega_{r}} \times$ $\mathbb{R}^{n} \backslash\{0\}$. We will distinguish those particular eigenvalues by rewriting them as $\lambda_{r k}^{+}(x, \xi)$ for $k=0, \ldots, n_{r}^{+}$and also let $J^{+}$be the set of $r, 0 \leq r \leq N_{0}$, such that there is a $j$ such that $\lambda_{j}(x, \xi) \in \Lambda^{+}$at some point $(x, \xi) \in \overline{\Omega_{r}} \times \mathbb{R}^{n} \backslash\{0\}$. Observing that similar results hold for the negative eigenvalues of $\omega(x)^{-1} \stackrel{\circ}{A}(x, \xi)$, let $J^{-}$be the set of $r, 0 \leq r \leq N_{0}$, such that there is a $j$ such that $\lambda_{j}(x, \xi) \in \Lambda^{-}$ 
at some point $(x, \xi) \in \overline{\Omega_{r}} \times \mathbb{R}^{n} \backslash\{0\}$, and let $n_{r}^{-}$and $\lambda_{r k}^{-}, k=0, \ldots, n_{r}^{-}$, be defined in a similar fashion to $n_{r}^{+}$and $\lambda_{r k}^{+}$. Lastly, for $q>n / 2 m$ let us denote by $\operatorname{tr}^{(+)} R(\lambda)^{q}$ that part of the trace of $R(\lambda)^{q}$ which corresponds to the eigenvalues of $V^{-1} A$ lying in $\Lambda^{+}$.

Theorem 4.6. Suppose that the boundary problem (1.1), (1.2) is weakly smooth and elliptic with parameter in the two closed sectors $\mathcal{L}^{(1)}$ and $\mathcal{L}^{(2)}$ cited above and that the assumptions made above concerning $\omega(x)^{-1} \stackrel{\circ}{A}(x, \xi)$ hold. Suppose also that $n / 2 m<q<n / 2 m+1$. Then

$$
\operatorname{tr}^{(+)} R(\lambda)^{q}=c_{q}^{+}(-\lambda)^{\frac{n}{2 m}-q}+o\left(|\lambda|^{\frac{n}{2 m}-q}\right) \text { as }|\lambda| \rightarrow \infty
$$

uniformly in any closed sector that has no common points with $\overline{\Lambda^{+}}$except 0 , where

$$
c_{q}^{+}=\frac{1}{(2 \pi)^{n}} \sum_{r \in J^{+}} \int_{\Omega_{r}} d x \sum_{k=1}^{n_{r}^{+}} \int_{\mathbb{R}^{n}}\left(\lambda_{r k}^{+}(x, \xi)+1\right)^{-q} d \xi
$$

and the power $(-\lambda) \cdots$ is defined by using a cut along $\overline{\mathbb{R}_{+}}$.

Proof. Recalling that each $\lambda_{j}(x, \xi)$ is continuous in $\overline{\Omega_{r}} \times \mathbb{R}^{n} \backslash\{0\}$ for $r=$ $0, \ldots, N_{0}$, it follows from Theorems 4.1, 4.5 and [5, Eq. (1.29)] that for $\lambda \in \mathcal{L}^{(1)}$,

$$
\operatorname{tr} R(\gamma \lambda)^{q}=c_{q}(-\gamma \lambda)^{\frac{n}{2 m}-q}+o\left(|\lambda|^{\frac{n}{2 m}-q}\right) \text { as }|\lambda| \rightarrow \infty
$$

uniformly in $\mathcal{L}^{(1)}$, where $\gamma$ denotes a constant satisfying $|\gamma|=1,0<|\arg \gamma|<\pi$

$$
c_{q}=\frac{b_{n / 2 m, q}}{(2 \pi)^{n} n} \sum_{r=0}^{N_{0}} \int_{\Omega_{r}} d x \sum_{j=1}^{N} \int_{|\xi|=1}\left(\gamma \lambda_{j}(x, \xi)\right)^{-n / 2 m} d S_{\xi},
$$

the power $(-\gamma \lambda) \cdots$ is defined by using a cut along $\overline{\mathbb{R}_{+}}$, and the power $\left(\gamma \lambda_{j}(x, \xi)\right) \cdots$ is defined by using a cut along $\overline{\mathbb{R}_{-}}$if $\lambda_{j}(x, \xi)>0$ or if $\lambda_{j}(x, \xi)<0$ and $\arg \gamma>0$, and along $\overline{\mathbb{R}_{+}}$if $\lambda_{j}(x, \xi)<0$ and arg $\gamma<0$. Now suppose that for some $r$, $0 \leq r \leq N_{0}, \lambda_{j}(x, \xi)>0$ for $(x, \xi) \in \overline{\Omega_{r}} \times \mathbb{R}^{n} \backslash\{0\}$. Then

$$
\begin{aligned}
& \frac{b_{n / 2 m, q}}{n}(-\gamma \lambda)^{\frac{n}{2 m}-q} \int_{|\xi|=1}\left(\gamma \lambda_{j}(x, \xi)\right)^{-n / 2 m} d S_{\xi} \\
& =\frac{b_{n / 2 m, q}}{n} \gamma^{-q}(-\lambda)^{\frac{n}{2 m}-q} \int_{|\xi|=1}\left(\lambda_{j}(x, \xi)\right)^{-n / 2 m} d S_{\xi} \\
& =\gamma^{-q}(-\lambda)^{\frac{n}{2 m}-q} \int_{\xi \in \mathbb{R}^{n}}\left(\lambda_{j}(x, \xi)+1\right)^{-q} d \xi,
\end{aligned}
$$

where the power $(-\lambda)^{\cdots}$ is defined by using a cut along $\overline{\mathbb{R}_{+}}$. On the other hand, if for some $r, 0 \leq r \leq N_{0}, \lambda_{j}(x, \xi)<0$ for $(x, \xi) \in \overline{\Omega_{r}} \times \mathbb{R}^{n} \backslash\{0\}$, then

$$
\begin{aligned}
& \frac{b_{n / 2 m, q}}{n}(-\gamma \lambda)^{\frac{n}{2 m}-q} \int_{|\xi|=1}\left(\gamma \lambda_{j}(x, \xi)\right)^{-n / 2 m} d S_{\xi} \\
& =\frac{b_{n / 2 m, q}}{n} e^{i \pi q} \gamma^{-q} \lambda^{\frac{n}{2 m}-q} \int_{|\xi|=1}\left|\lambda_{j}(x, \xi)\right|^{-n / 2 m} d S_{\xi} \\
& =e^{i \pi q} \gamma^{-q} \lambda^{\frac{n}{2 m}-q} \int_{\mathbb{R}^{n}}\left(\left|\lambda_{j}(x, \xi)\right|+1\right)^{-q} d \xi,
\end{aligned}
$$


where the power $(\lambda)^{\cdots}$ is defined by using a cut along $\overline{\mathbb{R}_{-}}$. Hence it follows that

$$
\operatorname{tr} R(\lambda)^{q}=c_{q}^{+}(-\lambda)^{\frac{n}{2 m}-q}+c_{q}^{-} \lambda^{\frac{n}{2 m}-q}+o\left(|\lambda| \frac{n}{2 m}-q\right) \text { as }|\lambda| \rightarrow \infty
$$

uniformly in $\mathcal{L}^{(1)}$, where

$$
c_{q}^{-}=\frac{e^{i \pi q}}{(2 \pi)^{n}} \sum_{r \in J^{-}} \int_{\Omega_{r}} d x \sum_{k=1}^{n_{r}^{-}}\left(\left|\lambda_{r k}^{-}(x, \xi)\right|+1\right)^{-q} d \xi
$$

and the powers are defined above. Since a similar result holds for $\lambda \in \mathcal{L}^{(2)}$, we conclude that (4.7) holds uniformly in $\mathcal{L}^{(1)} \cup \mathcal{L}^{(2)}$. In light of this fact we may now employ arguments similar to those used in the proofs of [5, Theorem 1.17], [6, Theorem 6.5] to complete the proof of the theorem.

We are now in a position to deal with the case where $\stackrel{\circ}{A}(x, \xi)$ is positive definite in $\bar{\Omega} \times \mathbb{R}^{n} \backslash\{0\}$ and for at least one $r, 0 \leq r \leq N_{0}$, the components of $\omega(x)$ are not all of the same sign in $\overline{\Omega_{r}}$. Fixing our attention upon this case, we observe from the proof of Proposition A.1 that for $x \in \overline{\Omega_{r}}, \omega(x)^{-1} \stackrel{\circ}{A}(x, \xi)$ has both positive and negative eigenvalues. Furthermore for $\theta \neq k \pi, k \in \mathbb{Z}$, Conditions 1 and 2 of Definition 2.3 are satisfied for $\mathcal{L}=\mathcal{L}(\theta)$, and hence in order that the boundary problem $(1.1),(1.2)$ be elliptic with parameter in $\mathcal{L}(\theta)$ we only require that Condition 2 be satisfied. Observe from Proposition A. 2 that this will always be the case if the boundary conditions (1.2) are of Dirichlet type. On the other hand, if $\theta=k \pi, k \in \mathbb{Z}$, then the boundary problem (1.1), (1.2) cannot be elliptic with parameter in $\mathcal{L}(\theta)$. Consequently it follows from Proposition 3.4 that for any $\varepsilon$ satisfying $0<\varepsilon<\pi / 2$, there are infinitely many eigenvalues of $V^{-1} A$ lying in each of the sectors $|\arg \lambda|<\varepsilon$ and $|\arg \lambda-\pi|<\varepsilon$, while there are at most a finite number of eigenvalues lying in each of the sectors $\varepsilon \leq \arg \lambda \leq \pi-\varepsilon$ and $-\pi+\varepsilon \leq \arg \lambda \leq-\varepsilon$. Lastly let $N^{+}(t)=\operatorname{card}\left\{j|| \lambda_{j} \mid \leq t, \operatorname{Re} \lambda_{j} \geq 0\right\}$, $N^{-}(t)=\operatorname{card}\left\{j|| \lambda_{j} \mid \leq t, \operatorname{Re} \lambda_{j}<0\right\}$, so that $N(t)=N^{+}(t)+N^{-}(t)$. Then recalling the definitions of the $J^{ \pm}, n_{r}^{ \pm}, \lambda_{r j}^{ \pm}$given in the statement preceding Theorem 4.6, we have next

Theorem 4.7. Suppose that $\stackrel{\circ}{A}(x, \xi)$ is positive definite in $\bar{\Omega} \times \mathbb{R}^{n} \backslash\{0\}$ and that for at least one $r, 0 \leq r \leq N_{0}$, the components of $\omega(x)$ are not all of the same sign in $\overline{\Omega_{r}}$. Suppose also that the boundary problem (1.1), (1.2) is weakly smooth and that Condition 2 of Definition 2.3 holds for every ray $\mathcal{L}(\theta)$ for which $\theta \neq k \pi$, $k \in \mathbb{Z}$. Then

$$
\begin{aligned}
N^{ \pm}(t) & =\kappa^{ \pm} t^{n / 2 m}+o\left(t^{n / m}\right) \text { as } t \rightarrow \infty, \\
N(t) & =\kappa t^{n / 2 m}+o\left(t^{n / m}\right) \text { as } t \rightarrow \infty,
\end{aligned}
$$

where

$$
\kappa^{+}=\frac{1}{(2 \pi)^{n}} \sum_{r \in J^{+}} \int_{\Omega_{r}} d x \sum_{k=1}^{n_{r}^{+}} \int_{\lambda_{r k}^{+}(x, \xi)<1} d \xi
$$




$$
\begin{aligned}
\kappa^{-} & =\frac{1}{(2 \pi)^{n}} \sum_{r \in J^{-}} \int_{\Omega_{r}} d x \sum_{k=1}^{n_{r}^{-}} \int_{\left|\lambda_{r k}^{-}(x, \xi)\right|<1} d \xi, \\
\kappa & =\frac{1}{(2 \pi)^{n}} \int_{\Omega} d x \sum_{j=1}^{N} \int_{\left|\lambda_{j}(x, \xi)\right|<1} d \xi .
\end{aligned}
$$

Proof. It follows from Theorem 4.6 that

$$
\sum_{\operatorname{Re} \lambda_{j} \geq 0} \frac{1}{\left(\lambda_{j}+t\right)^{q}}=b_{n / 2 m, q} d_{q}^{+} t^{\frac{n}{2 m}-q}+o\left(t^{\frac{n}{2 m}-q}\right) \text { as } t \rightarrow \infty,
$$

where $c_{q}^{+}=b_{n / 2 m, q} d_{q}^{+}$, and hence we obtain from arguments somewhat similar to those used in the proof of [6, Theorem 6.3] that

$$
\sum_{\operatorname{Re} \lambda_{j} \geq 0} \frac{1}{\left(\left|\lambda_{j}\right|+t\right)^{q}}=b_{b / 2 m, q} d_{q}^{+} t^{\frac{n}{2 m}-q}+o\left(t^{\frac{n}{m}-q}\right) \text { as } t \rightarrow \infty .
$$

The assertion for $N^{+}(t)$ now follows from the Hardy-Littlewood Tauberian theorem (see the proof of Theorem 4.4). The assertion for $N^{-}(t)$ can be obtained in the same way by replacing $\omega(x)$ by $-\omega(x)$. The assertion for $N(t)$ then follows from these results.

\section{Appendix A}

In this appendix we are going to prove various assertions made in Section 1.

Proposition A.1. Suppose that the boundary problem (1.1), (1.2) is minimally smooth and that Condition 1 of Definition 2.3 holds. Suppose also that $x^{0} \in \Gamma_{r}$, $1 \leq r \leq N_{0}$. If $\stackrel{\circ}{A}\left(x^{0}, \xi\right)$ is positive definite for $\xi \in \mathbb{R}^{n} \backslash\{0\}$, then Condition 3 of Definition 2.3 holds.

Proof. Rewriting the boundary problem (1.1), (1.4) in terms of the local coordinates at $x^{0}$ as in Condition 3 of Definition 2.3, let us note that our assumption concerning $\AA$ implies that $\langle\AA(0, \xi) z, z\rangle \geq c|\xi|^{2 m}|z|^{2}$ for $z \in \mathbb{C}^{N}$ and $\xi \in \mathbb{R}^{n}$, where $c$ denotes a positive constant and $\langle\cdot, \cdot\rangle$ the inner product in $\mathbb{C}^{N}$. Let us note in addition that if $\mu$ is an eigenvalue of $\omega^{+}(0)^{-1} \stackrel{\circ}{A}(0, \xi), \xi \in \mathbb{R}^{n} \backslash\{0\}$, and $z$ a corresponding eigenvector, then $\langle\stackrel{\circ}{A}(0, \xi) z, z\rangle=\mu\left\langle\omega^{+}(0) z, z\right\rangle$, which implies that if all the components of $\omega^{+}(0)$ have the same sign, then $\mu$ has the same sign also. On the other hand, if not all of the components of $\omega^{+}(0)$ have the same sign and if we equip $\mathbb{C}^{N}$ with the inner product $\langle\cdot, \cdot\rangle_{\omega^{+}}=\left\langle\omega^{+}(0) \cdot, \cdot\right\rangle$, then $\mathbb{C}^{N}$ becomes a finite dimensional Pontrjagin space and $\omega^{+}(0)^{-1} \stackrel{\circ}{A}(0, \xi)$ is a Pesonen operator in this space (see [7, p.48]). It follows from [7, Theorem 9.10, p.49] that in this case $\omega^{+}(0)^{-1} \AA(0, \xi)$ has both positive and negative eigenvalues. Thus we have shown that if all the components of $\omega^{+}(0)$ are of the same sign, say positive (resp. 
negative), then $\mathcal{L}$ intersects $\overline{\mathbb{R}_{+}}$(resp. $\overline{\mathbb{R}_{-}}$) only at the origin, while in the contrary case, $\mathcal{L}$ intersects $\mathbb{R}$ only at the origin. A similar result holds for $\omega^{-}(0)$ in place of $\omega^{+}(0)$.

Let $v^{+}(t)$ be a solution of (2.4) decaying exponentially at $\infty$ (see [3, Section $3]), v^{-}(t)$ a solution of (2.5) decaying exponentially at $-\infty$, and suppose that (2.6) holds. Hence if we put $v(t)=v^{+}(t)$ for $t>0, v(t)=v^{-}(t)$ for $t<0$, then it follows that $v \in W_{2}^{2 m}(\mathbb{R})$ and

$$
\left(\stackrel{\circ}{A}\left(0, \xi^{\prime}, D_{n}\right) v, v\right)_{\mathbb{R}}-\lambda\left[\left(\omega^{+}(0) v, v\right)_{\mathbb{R}_{+}}+\left(\omega^{-}(0) v, v\right)_{\mathbb{R}_{-}}\right]=0,
$$

where for $X$ a measurable subset of $\mathbb{R}$ we use the notation $(\cdot, \cdot)_{X}$ to denote the inner product in $L_{2}(X)^{N}$. Putting $\lambda=\mu+i \nu$, we obtain from (A.1) that

$$
\begin{aligned}
c\left(|\xi|^{2 m} \hat{v}, \hat{v}\right)_{\mathbb{R}^{-}}-\mu\left[\left(\omega^{+}(0) v, v\right)_{\mathbb{R}_{+}}+\left(\omega^{-}(0) v, v\right)_{\mathbb{R}_{-}}\right] \leq 0, \\
\nu\left[\left(\omega^{+}(0) v, v\right)_{\mathbb{R}_{+}}+\left(\omega^{-}(0) v, v\right)_{\mathbb{R}_{-}}\right]=0,
\end{aligned}
$$

where $\hat{v}$ denotes the Fourier transform of $v\left(t \rightarrow \xi_{n}\right)$. If $\lambda=0$ or if $\nu \neq 0$, then (A.2), (A.3) imply that $v=0$. On the other hand if $\nu=0$ and $\mu \neq 0$, then we know from above that the components of both $\omega^{+}(0)$ and $\omega^{-}(0)$ all have the same sign and that $\mu$ has opposite sign, and hence it follows from (A.2) that $v=0$. This completes the proof of the proposition.

When the boundary conditions (1.2) are of Dirichlet type, i. e., of the form $\partial_{\nu}^{l} u_{k}=0$ on $\Gamma$ for $l=0, \ldots, m-1, k=1, \ldots, N$, where $\partial_{\nu}$ denotes differentiation along the interior normal to $\Gamma$, then a minor modification of the arguments used in the above proof (see [3, Chapter 1]) gives the following analogue of Proposition A.1 for the boundary $\Gamma$.

Proposition A.2. Suppose that the boundary conditions (1.2) are of Dirichlet type. Suppose also that the boundary problem (1.1), (1.2) is minimally smooth and that Condition 1 of Definition (2.3) holds. Let $x^{0} \in \Gamma$. If $\AA\left(x^{0}, \xi\right)$ is positive definite for $\xi \in \mathbb{R}^{n} \backslash\{0\}$, then Condition 2 of Definition (2.3) holds.

Proposition A.3. Suppose that the boundary problem (1.1), (1.2) is minimally smooth and that Condition 1 of Definition 2.3 holds. Suppose also that $x^{0} \in \Gamma_{r}$, $1 \leq r \leq N_{0}$. If the matrix operator $\stackrel{\circ}{A}\left(x^{0}, D\right)$ is triangular, then Condition 3 of Definition 2.3 holds.

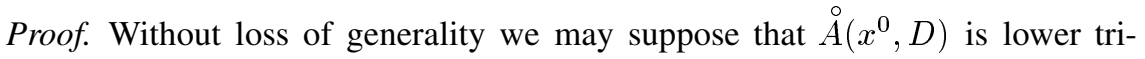
angular. Then rewriting the boundary problem (1.1), (1.4) in terms of the local coordinates at $x^{0}$ as in Condition 3 of Definition 2.3, let $v^{+}(t)$ be a solution of (2.4) decaying exponentially at $\infty, v^{-}(t)$ a solution of (2.5) decaying exponentially at $-\infty$, and suppose that (2.6) holds. Let $v(t)=v^{+}(t)$ for $t>0$, $v(t)=v^{-}(t)$ for $t<0$, and denote the components of $v(t)$ by $v_{j}(t), j=1, \ldots, N$. Then $v_{1}(t) \in W_{2}^{2 m}(\mathbb{R}),\left(\omega_{1}^{+}(0)^{-1} \AA_{11}\left(0, \xi^{\prime}, D_{n}\right)-\lambda\right) v_{1}(t)=0$ for $t>0$, and $\left(\omega_{1}^{-}(0)^{-1} \AA_{11}\left(0, \xi^{\prime}, D_{n}\right)-\lambda\right) v_{1}(t)=0$ for $t<0$, and hence it follows from [6, Section 7] that $v_{1}=0$. Arguing in the same way with $v_{2}, \ldots, v_{N}$, we arrive at the conclusion that $v=0$, which proves the proposition. 
Example A.4. We now give an example of a boundary problem which is minimally smooth and for which Conditions 1 and 2 of Definition 2.3 hold, but Condition 3 is violated. Accordingly, let us consider the boundary problem (1.1), (1.2) under the following conditions: (1) $N=2, m=N_{0}=1$; (2) $\Gamma$ and $\Gamma_{1}$ are of class $C^{1,1}$; (3) $\Omega_{1} \subset\left\{x \in \mathbb{R}^{n} \mid x_{n}>0\right\}$ and $\Gamma_{1}$ has, as part of its boundary, the set $\Gamma_{1}^{\prime}=\left\{x \in \mathbb{R}^{n}\left|x_{n}=0,\right| x^{\prime} \mid<\rho\right\}$, where $\rho>0$ and, putting $B_{\rho}(0)=\left\{x \in \mathbb{R}^{n}|| x \mid<\rho\right\}, \overline{B_{\rho}(0)} \cap \mathbb{R}_{+}^{n} \subset \Omega_{1}, \overline{B_{\rho}(0)} \cap \mathbb{R}_{-}^{n} \subset \Omega_{0} ;$ (4) $\mathcal{L}=i \overline{\mathbb{R}_{+}}$; (5) $A_{11}(x, D)=-\phi(x) \Delta, A_{12}(x, D)=\Delta, A_{21}(x, D)=$ $-(1-\phi(x)) \Delta, A_{22}(x, D)=-\phi(x) \Delta$, where $\Delta$ denotes the Laplacian in $\mathbb{R}^{n}$ and $\phi \in C_{0}^{\infty}(\Omega)$ such that $0 \leq \phi(x) \leq 1, \phi(x)=0$ for $|x|<\rho / 2, \phi(x)=1$ for $|x|>\rho$; (6) $B_{j k}(x, D)=\delta_{j k}$, where $\delta_{j k}$ denotes the Kronecker delta; (7) $\omega(x)$ satisfies the relevant conditions of Assumption 2.1, $\omega_{1}(x) \omega_{2}(x)<0$ for $x \in \overline{\Omega_{r}}, r=0,1$, and with $\omega^{+}(0)=\lim _{\substack{x \rightarrow 0 \\ x \in \Omega_{1}}} \omega(x)$ and $\omega^{-}(0)=\lim _{\substack{x \rightarrow 0 \\ x \in \Omega_{0}}} \omega(x)$, $\omega_{1}^{-}(0)=-\tau_{0}^{4} \omega_{1}^{+}(0)$ and $\omega_{2}^{-}(0)=-\omega_{2}^{+}(0)$, where $\tau_{0}$ is the root of the polynomial $P(\tau)=\tau^{4}-2 \tau^{3}-2 \tau^{2}-2 \tau+1$ lying between 0 and 1 .

It is clear that under our conditions the boundary problem (1.1), (1.2) is minimally smooth, while a direct calculation shows that Condition 1 of Definition 2.3 is satisfied. That Condition 2 is satisfied follows from [3, Section 3], while Proposition A. 3 shows that Condition 3 is satisfied at all points $x^{0} \in \Gamma_{1} \backslash \Gamma_{1}^{\prime}$. We now show that Condition 3 is not satisfied when $x^{0}=0, \xi^{\prime}=0$, and $\lambda=i$. To this end we observe that for this case (2.4), (2.5) become

$$
\begin{aligned}
& \stackrel{\AA}{A}\left(0,0, D_{n}\right) v^{+}(t)-i \omega^{+}(0) v^{+}(t)=0 \text { for } t>0, \\
& \stackrel{\circ}{A}\left(0,0, D_{n}\right) v^{-}(t)-i \omega^{-}(0) v^{-}(t)=0 \text { for } t<0,
\end{aligned}
$$

where

$$
\stackrel{\circ}{A}\left(0,0, D_{n}\right)=\left(\begin{array}{cc}
0 & -D_{n}^{2} \\
D_{n}^{2} & 0
\end{array}\right) .
$$

By reduction to triangular form as in [3], it is readily verified that (A.4) has precisely two linearly independent solutions decaying exponentially at $\infty$, namely

$$
u^{+}(t)=\left(\begin{array}{c}
-\rho^{+} \\
1
\end{array}\right) e^{i \alpha_{1}^{+} t} \text { and } \widetilde{u}^{+}(t)=\left(\begin{array}{c}
\rho^{+} \\
1
\end{array}\right) e^{i \alpha_{2}^{+} t},
$$

while (A.5) has precisely two linearly independent solutions decaying exponentially at $-\infty$, namely

$$
u^{-}(t)=\left(\begin{array}{c}
-\rho^{-} \\
1
\end{array}\right) e^{i \alpha_{1}^{-} t} \text { and } \widetilde{u}^{-}(t)=\left(\begin{array}{c}
\rho^{-} \\
1
\end{array}\right) e^{i \alpha_{2}^{-} t},
$$

where $\rho^{ \pm}=\left|\omega_{1}^{ \pm}(0) \omega_{2}^{ \pm}(0)\right|^{1 / 2} / \omega_{1}^{ \pm}(0), \alpha_{1}^{+}=-i \alpha_{2}^{+}=\delta^{+} e^{i \pi / 4}, \alpha_{1}^{-}=-i \alpha_{2}^{-}=$ $\delta^{-} e^{5 \pi i / 4}$, and $\delta^{ \pm}=\left|\omega_{1}^{ \pm}(0) \omega_{2}^{ \pm}(0)\right|^{1 / 4}$. Since we must have $v^{+}(t)=c_{1}^{+} u^{+}(t)+$ $c_{2}^{+} \widetilde{u}^{+}(t)$ for $t \geq 0$ and $v^{-}(t)=c_{1}^{-} u^{-}(t)+c_{2}^{-} \widetilde{u}^{-}(t)$ for $t \leq 0$ for some constants 
$c_{j}^{ \pm}$, we see that the boundary problem (A.4), (A.5), (2.6), (2.7) has only the trivial solution if and only if

$$
\operatorname{det}\left(\begin{array}{cccc}
-\rho^{+} & \rho^{+} & -\rho^{-} & \rho^{-} \\
-\rho^{+} \alpha_{1}^{+} & \rho^{+} \alpha_{2}^{+} & -\rho^{-} \alpha_{1}^{-} & \rho^{-} \alpha_{2}^{-} \\
1 & 1 & 1 & 1 \\
\alpha_{1}^{+} & \alpha_{2}^{+} & \alpha_{1}^{-} & \alpha_{2}^{-}
\end{array}\right) \neq 0,
$$

or equivalently (after some reduction), if and only if

$$
y_{1}^{4}+y_{2}^{4}-2 y_{1}^{3} y_{2}^{3}-2 y_{1}^{2} y_{2}^{2}-2 y_{1} y_{2} \neq 0,
$$

where $y_{j}=\left|\omega_{j}^{-}(0) / \omega_{j}^{+}(0)\right|^{1 / 4}$ for $j=1,2$. But since the expression on the left side of (A.6) is just $P\left(\tau_{0}\right)$, which is zero, we have thus shown that the problem (A.4), (A.5), (2.6), (2.7) admits a non-trivial solution.

Turning to the adjoint problem (1.5), (1.6), let us now show that if the system of boundary operators (1.4) covers the operator $A(x, D)-\lambda \omega(x)$ on $\Gamma_{r}$ for $r=$ $1, \ldots, N_{0}$ (in the sense described in Section 1), then it also covers the boundary operator $A^{\prime}(x, D)-\bar{\lambda} \omega(x)$ on the $\Gamma_{r}$. To this end let us denote by $\AA^{\prime}(x, \xi)$ the principal symbol of $A^{\prime}(x, D)$ (this is defined in an analogous manner to that for $A(x, D)$ ).

Proposition A.5. Suppose that the boundary problem (1.1), (1.2) is weakly smooth and elliptic with parameter in $\mathcal{L}$. Then Condition 1 of Definition 2.3 remains valid when $\stackrel{\AA}{A}(x, \xi)$ is replaced by $\AA^{\prime}(x, \xi)$ and $\lambda$ by $\bar{\lambda}$. Furthermore, if $x^{0} \in \Gamma_{r}, 1 \leq$ $r \leq N_{0}$, then Condition 3 of Definition 2.3 also remains valid when $\AA\left(0, \xi^{\prime}, D_{n}\right)$ is replaced by $\AA^{\prime}\left(0, \xi^{\prime}, D_{n}\right)$ and $\lambda$ by $\bar{\lambda}$.

Proof. The first assertion of the proposition is clear and so we henceforth fix our attention upon the second assertion. The idea now is to reduce the boundary problem (1.1), (1.4) to a boundary problem in $\Omega_{r}$ so that we can use the results of [12], [13] concerning the Green formula for such a boundary problem to prove the assertion.

By hypothesis there exists the diffeomorphism $\Phi$ of class $C^{2 m-1,1}$ mapping $\Gamma_{r} \times(-1,1)$ (the points of which are denoted by $x=\left(x^{\prime}, x_{n}\right)$ ) onto a tubular neighbourhood $\Sigma^{\prime}$ of $\Gamma_{r}$ in $\mathbb{R}^{n}$ with $\Gamma_{r} \times[0,1)$ being mapped onto the collar $\Sigma_{+}^{\prime}=\Sigma^{\prime} \cap \overline{\Omega_{r}}$ and $\Gamma \times(-1,0)$ onto $\Sigma_{-}^{\prime}=\Sigma^{\prime} \cap \Omega_{0}$. Thus we may identify $\Sigma_{+}^{\prime}$ with $\Gamma_{r} \times[0,1), \Gamma_{r}$ with $\Gamma_{r} \times\{0\}$, and $\Sigma_{-}^{\prime}$ with $\Gamma_{r} \times(-1,0)$, and hence if we put

$$
A_{\alpha}(x)=\left(a_{\alpha}^{j k}(x)\right)_{j, k=1}^{N} \text { for } 0 \leq|\alpha| \leq 2 m,
$$

then in $\Sigma^{\prime}$ we can write $A(x, D)$ in the form

$$
A(x, D)=\sum_{k=0}^{2 m} A_{k}\left(x, D^{\prime}\right) D_{n}^{k}
$$


where

$$
A_{k}\left(x, D^{\prime}\right)=\sum_{\substack{|\alpha| \geq k \\ \alpha_{n}=k}} A_{\alpha}(x) D^{\prime \alpha^{\prime}} .
$$

It is important to observe that $A_{2 m}\left(x, D^{\prime}\right)$ is a matrix of functions which is invertible in $\Sigma^{\prime}$ and which we henceforth denote by $A_{2 m}(x)$. For $x$ in the interior $\stackrel{\circ}{\Sigma}_{+}^{\prime}$ of $\Sigma_{+}^{\prime}$, let $\mathcal{A}_{k, \alpha}(x)=\operatorname{diag}\left(A_{\alpha}\left(x^{\prime}, x_{n}\right),(-1)^{k} A_{\alpha}\left(x^{\prime},-x_{n}\right)\right)$ for $0 \leq k,|\alpha| \leq 2 m$,

$$
\mathcal{A}_{k}\left(x, D^{\prime}\right)=\sum_{\substack{|\alpha| \geq k \\ \alpha_{n}=k}} \mathcal{A}_{k, \alpha}(x) D^{\prime \alpha^{\prime}}
$$

for $k=1, \ldots, 2 m$,

$$
\widetilde{\omega}(x)=\operatorname{diag}\left(\omega\left(x^{\prime}, x_{n}\right), \omega\left(x^{\prime},-x_{n}\right)\right), \quad \mathcal{A}_{0}\left(x, D^{\prime}\right)=\mathcal{A}_{0}^{\dagger}\left(x, D^{\prime}\right)-\lambda \widetilde{\omega}(x),
$$

where $\mathcal{A}_{0}^{\dagger}\left(x, D^{\prime}\right)$ is given by (A.10) with $k=0$, and let us extend the definitions of the $\mathcal{A}_{k}\left(x, D^{\prime}\right)$ to all of $\Sigma_{+}^{\prime}$ by continuity. Then we can write the boundary problem (1.1), (1.4) in an equivalent form, namely as a boundary problem in $\Omega_{r}$, which in $\stackrel{\circ}{\Sigma^{\prime}}$, has the form

$$
\begin{aligned}
\mathcal{A}(x, D) u(x) & =f(x) \text { in } \stackrel{\circ}{\Sigma^{\prime}}, \\
S_{\ell} D_{n}^{\ell} u(x) & =0 \text { at } x_{n}=0 \text { for } \ell=0, \ldots, 2 m-1,
\end{aligned}
$$

where $\mathcal{A}(x, D)=\sum_{k=0}^{2 m} \mathcal{A}_{k}(x, D) D_{n}^{k}$ and $S_{\ell}=\left(I-(-1)^{\ell} I\right)$. Now let us observe from [12, Subsection 1.2] that we can also write $\mathcal{A}(x, D)$ in the form $\mathcal{A}(x, D)=\sum_{k=0}^{2 m} D_{n}^{k} \widetilde{\mathcal{A}}_{k}\left(x, D^{\prime}\right)$, where

$$
\widetilde{\mathcal{A}}_{k}\left(x, D^{\prime}\right)=\sum_{\ell=k}^{2 m} c_{k, \ell} \sum_{\substack{|\alpha| \geq \ell \\ \alpha_{n}=\ell}}\left(D_{n}^{\ell-k} \mathcal{A}_{\ell, \alpha}(x)\right) D^{\prime \alpha^{\prime}}
$$

and the $c_{k, \ell}$ are integers with $c_{k, k}=1$, and hence it follows that if $u, v \in$ $\left(C^{2 m-1,1}\left(\Omega_{r}\right)\right)^{2 N}$ and the support of $u$ is contained in $\Sigma_{+}^{\prime}$ as well as in a small neighbourhood $U$ of some point $x^{0} \in \Gamma_{r}$, then the Green formula (see [12, Subsection 1.2], [13, Proposition 1.3.2, p.37]) can be written in the form

$$
\begin{aligned}
& \int_{\substack{\Sigma_{+}^{\prime} \\
\Sigma_{+}}}\langle\mathcal{A}(x, D) u, v\rangle_{2 N} d x-\int_{\mathcal{S}_{\Sigma_{+}^{\prime}}}\left\langle u, \mathcal{A}^{\prime}(x, D) v\right\rangle_{2 N} d x \\
& =\sum_{k=0}^{2 m-1} \int_{\Gamma_{r}}\left\langle\sum_{\ell=0}^{2 m-1-k} \mathcal{U}_{k \ell}\left(x^{\prime}, D^{\prime}\right) \gamma_{\ell} u, \gamma_{k} v\right\rangle_{2 N} d x^{\prime}
\end{aligned}
$$

where $\langle\cdot, \cdot\rangle_{2 N}$ denotes the inner product in $\mathbb{C}^{2 N}, \mathcal{A}^{\prime}(x, D)$ denotes the formal adjoint of $\mathcal{A}(x, D)$,

$$
\gamma_{\ell} u\left(x^{\prime}\right)=D_{n}^{\ell} u\left(x^{\prime}, 0\right), \quad \gamma_{k} v\left(x^{\prime}\right)=D_{n}^{k} v\left(x^{\prime}, 0\right),
$$


and

$$
\mathcal{U}_{k \ell}\left(x^{\prime}, D^{\prime}\right)=i \sum_{s=k+\ell+1}^{2 m} c_{k, \ell, s} \sum_{\substack{|\alpha| \geq s \\ \alpha_{n}=s}}\left(D_{n}^{s-\ell-k-1} \mathcal{A}_{s, \alpha}\left(x^{\prime}, 0\right)\right) D^{\prime \alpha^{\prime}},
$$

where the $c_{k, \ell, s}$ are integers with $c_{k, k, 2 m}=1$. Hence if for $\ell=0, \ldots,(2 m-1)$ we let $O$ denote the $N \times N$ zero matrix, put $S_{\ell}^{\prime}=(O I), C_{\ell}=\left(\begin{array}{c}I \\ O\end{array}\right)$, and $C_{\ell}^{\prime}=\left(\begin{array}{c}(-1)^{\ell} I \\ I\end{array}\right)$, then it follows from [13, Lemma 1.6.1, p.75] that the Green formula corresponding to the boundary problem (A.11), (A.12) is given by (A.13) with the expression on the right side of this equation replaced by

$$
\sum_{\ell=0}^{2 m-1} \int_{\Gamma_{r}}\left\langle S_{\ell} \gamma_{\ell} u, \widetilde{T}_{\ell} v\right\rangle_{N} d x^{\prime}+\sum_{\ell=0}^{2 m-1} \int_{\Gamma_{r}}\left\langle S_{\ell}^{\prime} \gamma_{\ell} u, T_{\ell} v\right\rangle_{N} d x^{\prime}
$$

where $\langle\cdot, \cdot\rangle_{N}$ denotes the inner product in $\mathbb{C}^{N}, T_{\ell}$ and $\widetilde{T}_{\ell}$ are matrix operators whose entries are differential operators of order not exceeding $2 m-1-\ell$ and which are defined by

$$
\begin{aligned}
T_{\ell}= & T_{\ell}\left(x^{\prime}, D\right) \\
= & -i\left((-1)^{\ell} I I\right) \sum_{k=\ell}^{2 m-1} \sum_{s=2 m+\ell-k}^{2 m} c_{k, \ell, s} \times \\
& \times \sum_{\substack{|\alpha| \geq s \\
\alpha_{n}=s s}} D^{\prime \alpha^{\prime}}\left(D_{n}^{s+k-\ell-2 m} \mathcal{A}_{s, \alpha}\right)^{*}\left(x^{\prime}, 0\right) D_{n}^{2 m-1-k},
\end{aligned}
$$

$\widetilde{T}_{\ell}=\widetilde{T}_{\ell}\left(x^{\prime}, D\right)$ is obtained from (A.15) by replacing $\left((-1)^{\ell} I I\right)$ by $(I O)$, and * denotes adjoint. Observe that the coefficients of the differential operators appearing in the entries of $T_{\ell}$ and $\widetilde{T}_{\ell}$ are of class $C^{\ell, 1}\left(\Gamma_{r}\right)$.

It is clear that the proof of the proposition will be complete if we can show that the two systems of boundary operators $\left\{T_{\ell}\right\}_{\ell=0}^{2 m-1}$ and $\left\{S_{\ell} D_{n}^{\ell}\right\}_{\ell=0}^{2 m-1}$ are equivalent in the sense that $T_{\ell} v=0$ on $\Gamma_{r}$ for $\ell=0, \ldots,(2 m-1)$ if and only if $S_{\ell} D_{n}^{\ell} v=0$ on $\Gamma_{r}$ for $\ell=0, \ldots,(2 m-1)$. To achieve this goal we shall proceed by induction. Accordingly, for $\ell=2 m-1$ we see that on $\Gamma_{r}, T_{2 m-1} v=i A_{2 m}^{*}\left(x^{\prime}, 0\right) S_{0} \gamma_{0} v$, and hence $T_{2 m-1} v=0$ on $\Gamma_{r}$ if and only if $S_{0} \gamma_{0} v=0$. Let us suppose next that $0<j \leq 2 m-1$ and that $T_{2 m-1-\ell} v=0$ on $\Gamma_{r}$ for $\ell=0, \ldots,(j-1)$ if and only if $S_{\ell} \gamma_{\ell} v=0$ for $\ell=0, \ldots,(j-1)$, and prove that the same result holds for $\ell=0, \ldots, j$. To this end let us observe from (A.15) and the definitions of the terms involved that on $\Gamma_{r}$,

$$
\begin{aligned}
& T_{2 m-1-j}\left(x^{\prime}, D\right) v \\
& =i(-1)^{j}\left[\sum_{k=2 m-j}^{2 m-1} \sum_{s=4 m-j-k-1}^{2 m} c_{k, 2 m-j-1, s} \sum_{\substack{|\alpha| \geq s \\
\alpha_{n}=s}} \sum_{\beta^{\prime} \leq \alpha^{\prime}}(-1)^{\left|\alpha^{\prime}-\beta^{\prime}\right|}\left(\begin{array}{c}
\alpha^{\prime} \\
\beta^{\prime}
\end{array}\right) \times\right.
\end{aligned}
$$




$$
\begin{aligned}
& \left.\times\left(D^{\prime \alpha^{\prime}-\beta^{\prime}} D_{n}^{s+j+k+1-4 m} A_{\alpha}\left(x^{\prime}, 0\right)\right)^{*} D^{\prime \beta^{\prime}} S_{2 m-k-1} \gamma_{2 m-k-1} v\right] \\
& +i(-1)^{j} A_{2 m}^{*}\left(x^{\prime}, 0\right) S_{j} \gamma_{j} v
\end{aligned}
$$

where $\left(\begin{array}{c}\alpha^{\prime} \\ \beta^{\prime}\end{array}\right)$ has its usual meaning and where we note that $2 m-1-\ell$ runs from $j-1$ to 0 as $k$ runs from $2 m-j$ to $2 m-1$. Consequently, if we suppose that $S_{\ell} \gamma_{\ell} v=0$ for $\ell=0, \ldots, j$, then our inductive hypothesis and (A.16) show that $T_{2 m-1-\ell} v=0$ on $\Gamma_{r}$ for $\ell=0, \ldots, j$. Conversely, if $T_{2 m-1-\ell} v=0$ on $\Gamma_{r}$ for $\ell=0, \ldots, j$, then again our inductive hypothesis and (A.16) show that $S_{\ell} \gamma_{\ell} v=0$ for $\ell=0, \ldots, j$. In light of the principle of induction, the proof of the proposition is complete.

Finally, still fixing our attention upon the adjoint problem, we shall say that the boundary problem (1.5), (1.6) is elliptic with parameter in $\mathcal{L}$ if the analogue of Condition (a) of Definition 2.2 holds for this problem also and if Conditions 1-3 of Definition 2.3 hold with $A(x, D), \lambda$, and the $B_{j}(x, D)$ there replaced by $A^{\prime}(x, D), \bar{\lambda}$, and the $B_{j}^{\prime}(x, D)$, respectively.

Proposition A.6. Suppose that the boundary problem (1.1), (1.2) is weakly smooth and elliptic with parameter in $\mathcal{L}$. Then the boundary problem (1.5), (1.6) is elliptic with parameter in $\mathcal{L}$.

Proof. We shall prove the proposition by again appealing to the results of [12], [13] as we did in the proof of Proposition A.5. Accordingly, there exists the diffeomorphism $\Phi$ of class $C^{2 m-1,1}$ mapping $\Gamma \times(-1,1)$ (the points of which are denoted by $x=\left(x^{\prime}, x_{n}\right)$ ) onto a tubular neighbourhood $\Sigma^{\prime}$ of $\Gamma$ in $\mathbb{R}^{n}$ with $\Gamma \times[0,1)$ being mapped onto the collar $\Sigma_{+}^{\prime}=\Sigma^{\prime} \cap \overline{\Omega_{0}}$ and $\Gamma \times(-1,0)$ onto $\Sigma_{-}^{\prime}=\Sigma^{\prime} \cap\left(\mathbb{R}^{n} \backslash \bar{\Omega}\right)$. Thus if we identify $\Sigma_{+}^{\prime}$ with $\Gamma \times[0,1), \Gamma$ with $\Gamma \times\{0\}$, and $\Sigma_{-}^{\prime}$ with $\Gamma \times(-1,0)$, then in $\Sigma_{+}^{\prime}$ we can write $A(x, D)$ in the form (A.8), where, with $A_{\alpha}(x)$ given by (A.7), $A_{k}\left(x, D^{\prime}\right)$ is given by (A.9). We observe that $A_{2 m}\left(x, D^{\prime}\right)$ is a matrix of functions which is invertible in $\Sigma_{+}^{\prime}$ and which we henceforth denote by $A_{2 m}(x)$. We can now argue as in the proof of Proposition A.5 to show that if $u, v \in C^{2 m-1,1}(\Omega)$ and the support of $u$ is contained in $\Sigma_{+}^{\prime}$ as well as in a small neighbourhood $U$ of some point $x^{0} \in \Gamma$, then the Green formula can be written in the form

$$
\begin{gathered}
\int_{\substack{\Sigma_{+} \\
\Sigma_{+}^{\prime}}}\langle(A(x, D)-\lambda \omega(x)) u, v\rangle d x-\int_{\substack{\Sigma^{\prime} \\
\Sigma_{+}}}\left\langle u,\left(A^{\prime}(x, D)-\bar{\lambda} \omega(x)\right) v\right\rangle d x \\
=\sum_{r=0}^{2 m-1} \int_{\Gamma}\left\langle\gamma_{r} u, Z_{r}\left(x^{\prime}, D\right) v\right\rangle d x^{\prime}
\end{gathered}
$$

where $\stackrel{\circ}{\Sigma}_{+}^{\prime}$ denotes the interior of $\Sigma_{+}^{\prime},\langle\cdot, \cdot\rangle$ denotes the inner product in $\mathbb{C}^{N}, \gamma_{r} u$ is given by (A.14),

$$
Z_{r}\left(x^{\prime}, D\right)=-i \sum_{k=r}^{2 m-1} \sum_{s=2 m+r-k}^{2 m} c_{r, k, s} \times
$$




$$
\times \sum_{\substack{|\alpha| \geq s \\ \alpha_{n} \equiv s}} D^{\alpha^{\prime}}\left(D_{n}^{s+k-r-2 m} A_{\alpha}\left(x^{\prime}, 0\right)\right)^{*} D_{n}^{2 m-1-k},
$$

the $c_{r, k, s}$ are integers, and ${ }^{*}$ denotes adjoint.

By using a standard extension procedure we can suppose that the coefficients of the $B_{j k}(x, D)$, the $b_{\alpha}^{j k}(x, D)$, are defined in $\Sigma_{+}^{\prime}$ and that for each pair $j, k, b_{\alpha}^{j k} \in$ $C^{2 m-1-m_{j}, 1}\left(\Sigma_{+}^{\prime}\right) \cap C^{|\alpha|, 1}\left(\Sigma_{+}^{\prime}\right)$ for $|\alpha| \leq m_{j}$ (see Definition 2.2). Furthermore, we can suppose that

$$
0 \leq m_{1}=\cdots=m_{p_{1}}<m_{p_{1}+1}=\cdots=m_{p_{2}}<\cdots<m_{p_{q-1}+1}=\cdots=m_{p_{q}},
$$

where $p_{q}=m N$. Then putting $p_{0}=0, \widetilde{m}_{\ell}=m_{p_{\ell}}$,

$$
\mathfrak{B}_{\alpha}^{\left(\widetilde{m}_{\ell}\right)}(x)=\left(b_{\alpha}^{j k}(x)\right)_{\substack{j=p_{\ell-1}+1, \ldots, p_{\ell} \\ k=1, \ldots, N}} \text { for } 0 \leq|\alpha| \leq \widetilde{m}_{\ell},
$$

and

$$
\mathfrak{B}_{k}^{\left(\tilde{m}_{\ell}\right)}\left(x, D^{\prime}\right)=\sum_{\substack{k \leq|\alpha| \leq \tilde{m}_{\ell} \\ \alpha_{n}=k}} \mathfrak{B}_{\alpha}^{\left(\tilde{m}_{\ell}\right)}(x) D^{\prime \alpha^{\prime}} \text { for } 0 \leq k \leq \widetilde{m}_{\ell},
$$

$\ell=1, \ldots, q$, we see that in $\Sigma_{+}^{\prime}$ the boundary operators $\left\{B_{j}(x, D)\right\}_{1}^{m N}$ can be arranged in block form, namely

$$
\mathfrak{B}^{\left(\widetilde{m}_{\ell}\right)}(x, D)=\sum_{k=0}^{\widetilde{m}_{\ell}} \mathfrak{B}_{k}^{\left(\tilde{m}_{\ell}\right)}\left(x, D^{\prime}\right) D_{n}^{k}, \quad \ell=1, \ldots, q .
$$

It is important to observe that for each $\ell, \mathfrak{B}_{\widetilde{m}_{\ell}}^{\left(\widetilde{m}_{\ell}\right)}\left(x, D^{\prime}\right)$ is a matrix of functions which we henceforth denote by $\mathfrak{B}_{\tilde{m}_{\ell}}^{\left(\widetilde{m}_{\ell}\right)}(x)$. Furthermore, since it follows from Condition 2 of Definition 2.3 and [3, Theorem 3.2] that $\left\{B_{j}(x, D)\right\}_{1}^{m N}$ is a normal system of boundary operators, we conclude that $\mathfrak{B}_{\tilde{m}_{\ell}}^{\left(\tilde{m}_{\ell}\right)}\left(x^{\prime}, 0\right)$ has rank $n_{\ell}=\left(p_{\ell}-p_{\ell-1}\right) \leq N$ for $\ell=1, \ldots, q$.

We now introduce the block triangular matrix $\widetilde{S}\left(x^{\prime}, D^{\prime}\right)=\left(\widetilde{S}_{r k}\left(x^{\prime}, D^{\prime}\right)\right)_{r, k=0}^{2 m-1}$ on $\Gamma \cap U$, where the entries $\widetilde{S}_{r k}\left(x^{\prime}, D^{\prime}\right)$ denote $N \times N$ matrices of boundary operators, in the following way:

1 . if $r<2 m-1$ and $k>r$, then let $\widetilde{S}_{r k}\left(x^{\prime}, D^{\prime}\right)=O$, where $O$ denotes the $N \times N$ zero matrix;

2. if $r \neq \widetilde{m}_{\ell}$ for $\ell=1, \ldots, q$, then let $\widetilde{S}_{r k}\left(x^{\prime}, D^{\prime}\right)=O$ for $k=0, \ldots,(r-1)$, $\widetilde{S}_{k k}\left(x^{\prime}, D^{\prime}\right)=\widetilde{S}_{k k}\left(x^{\prime}\right)=I$;

3. if $r=\widetilde{m}_{\ell}$ for some $\ell$ and $n_{\ell}=N$, then let $\widetilde{S}_{\widetilde{m}_{\ell}, k}\left(x^{\prime}, D^{\prime}\right)=\mathfrak{B}_{k}^{\left(\widetilde{m}_{\ell}\right)}\left(x^{\prime}, 0, D^{\prime}\right)$ for $k=0, \ldots,\left(\widetilde{m}_{\ell}-1\right), \widetilde{S}_{\widetilde{m}_{\ell}, \widetilde{m}_{\ell}}\left(x^{\prime}, D^{\prime}\right)=\widetilde{S}_{\widetilde{m}_{\ell}, \widetilde{m}_{\ell}}\left(x^{\prime}\right)=\mathfrak{B}_{\widetilde{m}_{\ell}}^{\left(\widetilde{m}_{\ell}\right)}\left(x^{\prime}, 0\right)$;

4. if $r=\widetilde{m}_{\ell}$ for some $\ell$ and $n_{\ell}<N$, then let $\widetilde{S}_{\widetilde{m}_{\ell}, k}\left(x^{\prime}, D^{\prime}\right)=\left(\begin{array}{c}\mathfrak{B}_{k}^{\left(\widetilde{m}_{\ell}\right)}\left(x^{\prime}, 0, D^{\prime}\right) \\ O_{\widetilde{m}_{\ell}}\end{array}\right)$ 
for $k=0, \ldots,\left(\widetilde{m}_{\ell}-1\right), \widetilde{S}_{\widetilde{m}_{\ell}, \widetilde{m}_{\ell}}\left(x^{\prime}, D^{\prime}\right)=\widetilde{S}_{\widetilde{m}_{\ell}, \widetilde{m}_{\ell}}\left(x^{\prime}\right)=\left(\begin{array}{c}\mathfrak{B}_{\widetilde{m}_{\ell}}^{\left(\widetilde{m}_{\ell}\right)}\left(x^{\prime}, 0\right) \\ I_{\widetilde{m}_{\ell}}\end{array}\right)$, where $O_{\widetilde{m}}$ and $I_{\widetilde{m}_{\ell}}$ denote $\left(N-n_{\ell}\right) \times N$ matrices with the entries of $O_{\widetilde{m}_{\ell}}$ being all zero while each row of $I_{\widetilde{m}_{\ell}}$ has precisely one non-zero entry, namely 1 , and $\operatorname{det} \widetilde{S}_{\widetilde{m}_{\ell}, \tilde{m}_{\ell}}\left(x^{\prime}\right) \neq 0$ for $x^{\prime} \in \Gamma \cap U$.

As in [13, Lemma 1.6.1, p.75] we also introduce the block triangular matrix $\widetilde{C}\left(x^{\prime}, D^{\prime}\right)=\left(\widetilde{C}_{r k}\left(x^{\prime}, D^{\prime}\right)\right)_{r, k=0}^{2 m-1}$ on $\Gamma \cap U$ satisfying $\widetilde{C}\left(x^{\prime}, D^{\prime}\right) \widetilde{S}\left(x^{\prime}, D^{\prime}\right)=$ $\operatorname{diag}(I, \ldots, I)$ (there are $2 m$ diagonal entries), where:

1. $\widetilde{C}_{r k}\left(x^{\prime}, D^{\prime}\right)=O$ if $r<2 m-1$ and $k>r$;

2. $\widetilde{C}_{r r}\left(x^{\prime}, D^{\prime}\right)=\widetilde{C}_{r r}\left(x^{\prime}\right)=\widetilde{S}_{r r}\left(x^{\prime}\right)^{-1}$ for $r=1, \ldots,(2 m-1)$;

3. if $1 \leq r \leq 2 m-1,0 \leq k<r$, and $r \neq \widetilde{m}_{\ell}$ for $\ell=1, \ldots, q$, then $\widetilde{C}_{r k}\left(x^{\prime}, \bar{D}^{\prime}\right)=O$;

4. if $1 \leq r \leq 2 m-1,0 \leq k<r$, and $r=\widetilde{m}_{\ell}$ for some $\ell$, then

$$
\begin{aligned}
\widetilde{C}_{\widetilde{m}_{\ell}, k}\left(x^{\prime}, D^{\prime}\right)= & \sum_{\beta} \delta_{\beta} \widetilde{S}_{\widetilde{m}_{\ell}, \widetilde{m}_{\ell}}\left(x^{\prime}\right)^{-1} \times \\
& \times \prod_{j=0}^{s} \widetilde{S}_{\widetilde{m}_{\beta_{j}}, \widetilde{m}_{\beta_{j+1}}}\left(x^{\prime}, D^{\prime}\right) \widetilde{S}_{\widetilde{m}_{\beta_{j+1}}, \widetilde{m}_{\beta_{j+1}}}\left(x^{\prime}\right)^{-1},
\end{aligned}
$$

where the summation is over all multi-indicies $\beta=\left(\beta_{0}, \ldots, \beta_{s}\right)$ satisfying $\ell=$ $\beta_{0}>\beta_{1}>\cdots>\beta_{s}$ with $\widetilde{m}_{\beta_{s}}>k, \widetilde{m}_{\beta_{s+1}}=k$, and $\delta_{\beta}$ is an integer. Hence the expression on the right side of the Green formula (A.17) can now be written as

$$
\begin{aligned}
\sum_{r}{ }_{\Gamma} \int_{\Gamma}\left\langle\gamma_{r} u, T_{r} v\right\rangle d x^{\prime} & +\sum_{\ell=1}^{q} \int_{\Gamma}\left\langle\sum_{k=0}^{\tilde{m}_{\ell}-1}\left(\begin{array}{c}
\mathfrak{B}_{k}^{\left(\widetilde{m}_{\ell}\right)}\left(x^{\prime}, D^{\prime}\right) \\
O_{\widetilde{m}_{\ell}}
\end{array}\right) \gamma_{k} u\right. \\
& \left.+\left(\begin{array}{c}
\mathfrak{B}_{\widetilde{m}_{\ell}\left(\widetilde{m}_{\ell}\right)}\left(x^{\prime}\right) \\
I_{\widetilde{m}_{\ell}}
\end{array}\right) \gamma_{\widetilde{m}_{\ell}} u, T_{\widetilde{m}_{\ell}} v\right\rangle d x^{\prime},
\end{aligned}
$$

where the first summation is over those $r, 0 \leq r \leq 2 m-1$, for which $r \neq \widetilde{m}_{\ell}$ for $\ell=1, \ldots, q$, in the second summation no $O_{\widetilde{m}_{\ell}}$ and $I_{\widetilde{m}_{\ell}}$ appear if $n_{\ell}=N$, and for $0 \leq \nu \leq 2 m-1$,

$$
\begin{aligned}
& T_{\nu}=T_{\nu}\left(x^{\prime}, D\right)=\widetilde{S}_{\nu \nu}^{*}\left(x^{\prime}\right)^{-1} Z_{\nu}\left(x^{\prime}, D\right)+\sum_{\substack { \tilde{m}_{\ell}>\nu \\
\begin{subarray}{c}{\beta=\ell \\
\tilde{m}_{\beta_{s+1}}=\nu{ \tilde { m } _ { \ell } > \nu \\
\begin{subarray} { c } { \beta = \ell \\
\tilde { m } _ { \beta _ { s + 1 } } = \nu } }\end{subarray}} \sum_{\beta} \times
\end{aligned}
$$

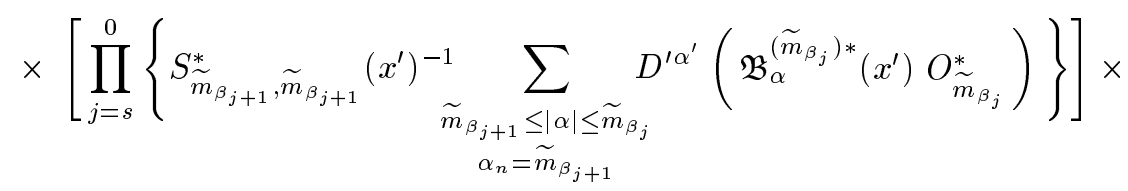

$$
\begin{aligned}
& \times S_{\tilde{m}_{\ell}, \tilde{m}_{\ell}}^{*}\left(x^{\prime}\right)^{-1} Z_{\widetilde{m}_{\ell}}\left(x^{\prime}, D\right),
\end{aligned}
$$

where here the summation does not appear if $\widetilde{m}_{q} \leq \nu$ and $O_{\widetilde{m}_{\beta_{j}}}^{*}$ does not appear if $n_{\beta_{j}}=N$. 
Inspection shows that the entries of $T_{\nu}\left(x^{\prime}, D\right)$ are differential operators of order not exceeding $2 m-1-\nu$ whose coefficients are of class $C^{\nu, 1}(\Gamma \cap U)$ and that the matrix $\left(T_{1}\left(x^{\prime}, D\right), \ldots, T_{2 m-1}\left(x^{\prime}, D\right)\right)^{T}$ is a Dirichlet matrix of order $(2 m, N)$ on $\Gamma \cap U$ in the sense of [16]. Since the $B_{j}^{\prime}(x, D)$ of (1.6) are precisely the rows of the $T_{r}$ in the first summation of (A.18) and the last $N-n_{\ell}$ rows of those $T_{\widetilde{m}_{\ell}}$ in the second summation for which $n_{\ell}<N$ (if any), it is clear from the definitions of the terms involved that the boundary problem (1.5), (1.6) satisfies the conditions of minimal smoothness. Finally, since it is also clear that the analogues of Conditions 1 and 2 of Definition 2.3 hold for the problem (1.5), (1.6), while the analogue of Condition 3 follows from Proposition A.5, the proof of the proposition is complete.

Acknowledgements. The second and the third authors were supported in part by the John Knopfmacher Centre for Applicable Analysis and Number Theory, University of the Witwatersrand. The third author was also supported in part by a grant from the NRF of South Africa.

\section{References}

[1] R. A. Adams, Sobolev Spaces, Academic Press, New York, 1975.

[2] S. Agmon, On the eigenfunctions and on the eigenvalues of general elliptic boundary value problems, Comm. Pure Appl. Math. 15 (1962), 119-147.

[3] S. Agmon, A. Douglis and L. Nirenberg, Estimates near the boundary for solutions of elliptic partial differential equations satisfying general boundary conditions II, Comm. Pure Appl. Math. 17 (1964), 35-92.

[4] M.S. Agranovich, Elliptic operators on closed manifolds, Encyclopaedia of Mathematical Sciences, vol. 63, pp. 1-130, Springer, Berlin, 1996.

[5] M.S. Agranovich and A. S. Markus, On the spectral properties of elliptic pseudodifferential operators far from selfadjoint ones, Z. Analysis. Anwend. 8 (1989), 237-260.

[6] M.S. Agranovich, R. Denk and M. Faierman, Weakly smooth nonselfadjoint spectral elliptic boundary problems, Spectral Theory, Microlocal Analysis, Singular Manifolds: Advances in Partial Differential Equations 14, 138-199 (Akademie-Verlag, Berlin, 1997).

[7] J. Bognár, Indefinite Inner Product Spaces, Springer, New York, 1974.

[8] N. Dunford and J. T. Schwartz, Linear Operators, Part II: Spectral theory, Wiley, New York, 1963.

[9] G. Geymonat, Su alcuni problemi ai limiti per i sistemi lineari ellittici secondo Petrowsky, Matematiche (Catania) 20 (1965), 211-253.

[10] I. C. Gohberg and M. G. Krein, Introduction to the Theory of Linear Nonselfadjoint Operators, Amer. Math. Soc., Providence, R.I., 1969.

[11] P. Grisvard, Elliptic Problems in Nonsmooth Domains, Pitman, London, 1985.

[12] G. Grubb, Boundary problems for systems of partial differential operators of mixed order, J. Functional Analysis 26 (1977), 131-165.

[13] G. Grubb, Functional Calculus of Pseudodifferential Boundary Problems, 2nd edn., Birkhäuser, Boston, 1996.

[14] T. Kato, Perturbation Theory for Linear Operators, 2nd edn., Springer, Berlin, 1976.

[15] J.L. Lions and E. Magenes, Non-homogeneous Boundary Value Problems and Applications, I, Springer, Berlin, 1972. 
[16] Ja.A. Roitberg and Z.G. Seftel, A theorem on homeomorphisms for elliptic systems and its applications, Math. USSR Sb. 7 (1969), 439-465.

[17] M. Sango, A spectral problem with an indefinite weight for an elliptic system, Elec. J. Diff. Eq. 21 (1997), 1-14.

[18] M. Sango, Asymptotic formulas for eigenvalues for an elliptic problem with indefinite weight, Appl. Anal. 72 (1999), 353-367.

[19] M. A. Shubin, Pseudodifferential Operators and Spectral Theory, Springer, Berlin, 1987.

[20] H. Triebel, Interpolation Theory, Function Spaces, Differential Operators, NorthHolland, Amsterdam, 1978.

[21] L. R. Volevich, Solvability of boundary value problems for general elliptic systems, Amer. Math. Soc. Transl., Ser. 2, 67 (1968), 182-225. 